\title{
A LOWER CENTRAL SERIES FOR SPLIT HOPF ALGEBRAS WITH INVOLUTION
}

BY

BRUCE W. JORDAN

\begin{abstract}
A lower central series is defined for split Hopf algebras with involution over a field $k$. Various structure theorems for coalgebras and Hopf algebras are established.
\end{abstract}

Introduction. In [6], Moore and Smith define a lower central series for homology Hopf algebras, i.e. connected Hopf algebras with commutative comultiplication. In essence their definition is a categorical one, the point being that the category of homology Hopf algebras possesses enough structure to apply the categorical construction.

It is the object of this paper to show that a lower central series can likewise be defined for objects of $\mathcal{H} / k$, the category of split Hopf algebras with involution over a field $k$. In order to accomplish this task it is necessary to expend much effort establishing the basic properties of $\mathcal{K} / k$. $\S 1$ lays the foundation for studying split Hopf algebras with involution by developing important results concerning coalgebras and comodules. $\S \S 2$ and 3 present structure theorems for objects in $\mathcal{K} / k$. The proof of the fact that epimorphisms are normal in $\mathcal{K} / k$ occupies all of $\S 4$. Finally in $\$ 5$ we are in a position to define the commutator sub-Hopf algebra of an object of $\mathcal{H} / k$ and to study descending series.

It is assumed that the reader is familiar with the work of Milnor and Moore [5]. Results and constructions from [5] are utilized freely throughout this paper. Also we use the terms Hopf algebra, coalgebra, comodule, etc., in the sense of Milnor and Moore, and we follow their notation whenever possible.

ACKNOWLEDGEMENTS. This paper is essentially the author's undergraduate thesis which was submitted to Princeton University in May, 1976. Professor John C. Moore was the faculty advisor for this work. He suggested many of the problems considered here and offered both encouragement and mathematical insight generously. The author's personal indebtedness to Professor Moore is great; the extent of his mathematical indebtedness should be apparent.

1. Coalgebras and comodules. In this section some elementary properties of coalgebras and comodules are established. In the first part of the section, we assume that a commutative ring $K$ has been chosen for the ground ring and tensor products are taken over $K$. $\Re / K$ will be used to denote the category of graded $K$-modules. In the later portion of the section the ground ring is usually a field $k$.

Received by the editors September 28, 1978.

AMS (MOS) subject classifications (1970). Primary 16A24. 
We begin with some categorical considerations. Let $\mathscr{D}$ be a category with point *. For $D^{\prime}, D^{\prime \prime} \in$ obj $\mathscr{D}$, let * denote the trivial morphism $D^{\prime} \rightarrow * \rightarrow D^{\prime \prime}$. Suppose $s: D^{\prime} \rightarrow D^{\prime \prime}$ is a morphism in $\mathscr{D}$. Then $\xi: N \rightarrow D^{\prime}$ is a kernel of $s$ if $s \circ \xi=*$, and whenever $g: F \rightarrow D^{\prime}$ is a morphism in $\mathscr{D}$ such that $s \circ g=*$ then there is a unique $\bar{g}: F \rightarrow N$ such that $\xi \circ \bar{g}=g$. Similarly if $s: D^{\prime} \rightarrow D^{\prime \prime}$ is a morphism in $\mathscr{D}$, then $\zeta: D^{\prime \prime} \rightarrow G$ is a cokernel of $s$ if $\zeta \circ s=*$ and whenever $h: D^{\prime \prime} \rightarrow F$ is a morphism in $\mathscr{D}$ such that $h \circ s=*$ there is a unique $\bar{h}: G \rightarrow F$ such that $h=\bar{h} \circ \zeta$. Kernels and cokernels are unique up to canonical isomorphism if they exist. (The above definitions are as given in [6].)

Let $A$ be an algebra over $K$ and let $B, B^{\prime \prime}$ be left $A$-modules. Suppose $f: B \rightarrow B^{\prime \prime}$ is a morphism of left $A$-modules. Let $B^{\prime}=\{x \in B \mid f(x)=0\}$ and $i: B^{\prime} \rightarrow B$ be the natural inclusion. $B^{\prime}$ is a $K$-submodule of $B$, and $i: B^{\prime} \rightarrow B$ is the kernel of $f$ : $B \rightarrow B^{\prime \prime}$ in $\Re / K$. We have the diagram

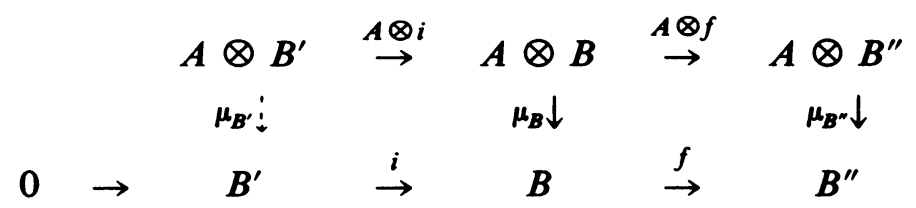

The right square commutes by the assumption that $f$ is a morphism of left $A$-modules. Observe that

$$
\mu_{B}(A \otimes i)\left(A \otimes B^{\prime}\right) \subseteq i\left(B^{\prime}\right)
$$

because

$$
f\left(\mu_{B}(A \otimes i)\right)=\mu_{B^{\prime \prime}}(A \otimes f)(A \otimes i)=0 .
$$

Define $\mu_{B^{\prime}}$ to be $i^{-1}\left(\mu_{B}(A \otimes i)\right)$. $\mu_{B^{\prime}}$ provides $B^{\prime}$ with a left $A$-module structure such that $i: B^{\prime} \rightarrow B$ is a morphism of left $A$-modules. $i: B^{\prime} \rightarrow B$ is in fact the kernel of $f$ : $B \rightarrow B^{\prime \prime}$ in ${ }_{A}$ Th, the category of left $A$-modules.

Similarly, if $f^{\prime}: B^{\prime} \rightarrow B$ is a morphism of left $A$-modules, let $\pi: B \rightarrow B^{\prime \prime}$ be the cokernel of $f^{\prime}: B^{\prime} \rightarrow B$ in $\mathfrak{T} / K$. Then $B^{\prime \prime}$ may be given the structure of a left $A$-module such that $\pi: B \rightarrow B^{\prime \prime}$ is a morphism of left $A$-modules by means of the diagram

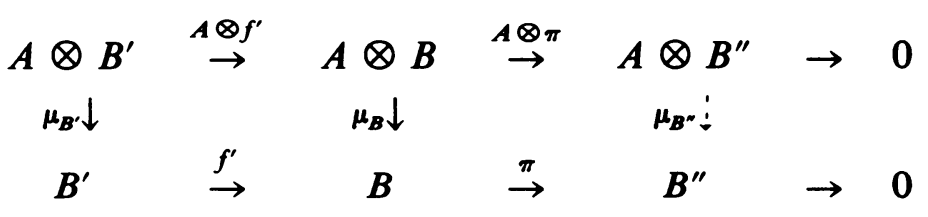

$\pi: B \rightarrow B^{\prime \prime}$ is the cokernel of $f$ in ${ }_{A} \mathfrak{T}$. These results partially verify the fact that $A_{A} \mathfrak{T}$ is an abelian category.

Now suppose $C$ is a coalgebra, and $B^{\prime}, B$ are right $C$-comodules. Let $f^{\prime}: B^{\prime} \rightarrow B$ be a morphism of right $C$-comodules and $\pi: B \rightarrow B^{\prime \prime}$ be the cokernel with respect to the graded $K$-module structure. Define a right $C$-comodule structure on $B^{\prime \prime}$ such that $\pi: B \rightarrow B^{\prime \prime}$ is a morphism of right $C$-comodules using the diagram 


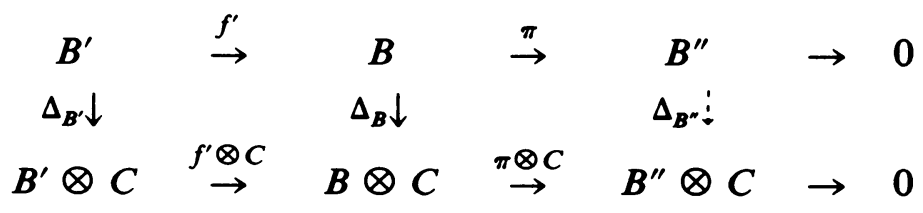

Hence cokernels exist in $(\bigodot \Re)_{C}$, the category of right $C$-comodules. However, kernels do not necessarily exist in $(\mathcal{C} \Re)_{C}$. The additional assumption that $C$ is flat as a graded $K$-module is needed to insure the existence of kernels. If $B$ and $B^{\prime \prime}$ are right $C$-comodules and $f: B \rightarrow B^{\prime \prime}$ is a morphism of right $C$-comodules, let $i$ : $B^{\prime} \rightarrow B$ be the kernel of $f$ in $\mathfrak{T} / K$. Assuming $C$ is flat we have the following diagram from which a right $C$-comodule structure can be defined on $B^{\prime}$ such that $i$ : $B^{\prime} \rightarrow B$ is a morphism of right $C$-comodules:

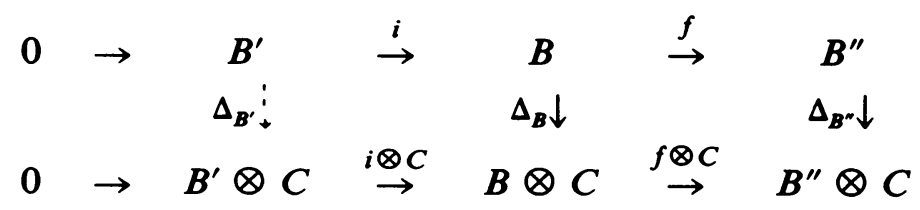

1.1. Proposition. Let $L$ be a graded $K$-module and $C$ a coalgebra over $K$. Suppose $M$ is a right $C$-comodule. Then

$$
\operatorname{Hom}_{C \text {-comodule }}(M, L \otimes C) \approx \operatorname{Hom}_{K}(M, L) .
$$

Proof. Suppose $f: M \rightarrow L$ is a morphism of graded $K$-modules. Define $\eta(f)$ : $M \rightarrow L \otimes C$ to be the composite

$$
\begin{aligned}
& M \stackrel{\Delta_{M}}{\rightarrow} M \otimes C \stackrel{f \otimes C}{\rightarrow} L \otimes C . \\
& (\eta(f) \otimes C) \Delta_{M}=\left((f \otimes C) \Delta_{M} \otimes C\right) \Delta_{M}=(f \otimes C \otimes C)\left(\Delta_{M} \otimes C\right) \Delta_{M}, \\
& \left(\Delta_{L \otimes C}\right)(\eta(f))=\left(L \otimes \Delta_{C}\right)(f \otimes C) \Delta_{M}=(f \otimes C \otimes C)\left(M \otimes \Delta_{C}\right) \Delta_{M} .
\end{aligned}
$$

Since $M$ is a right $C$-comodule, $\left(\Delta_{M} \otimes C\right) \Delta_{M}=\left(M \otimes \Delta_{C}\right) \Delta_{M}$. Hence $(\eta(f) \otimes$ C) $\Delta_{M}=\Delta_{L \otimes C} \eta(f)$ and $\eta(f)$ is a map of right $C$-comodules.

$$
\eta: \operatorname{Hom}_{K}(M, L) \rightarrow \operatorname{Hom}_{C \text {-comodules }}(M, L \otimes C)
$$

is easily seen to be a morphism of $K$-modules.

On the other hand, suppose $g: M \rightarrow L \otimes C$ is a morphism of right $C$-comodules. Then we have the mapping of graded $K$-modules $\Psi(f): M \rightarrow L$ by $M \stackrel{g}{\rightarrow} L \otimes C \stackrel{L \otimes \varepsilon}{\rightarrow} L \otimes K \approx L$. A routine verification shows that

$$
\eta \Psi=\operatorname{Hom}_{C \text {-comodule }}(M, L \otimes C) \text { and } \Psi \eta=\operatorname{Hom}_{K}(M, L) \text {. }
$$

Let $C$ be a coalgebra over $K$ which is flat as a graded $K$-module, and let $M$ be a right $C$-comodule. Suppose $x$ is an element of $M$ and $\Delta_{M} x=\Sigma m_{i} \otimes c_{i}$. Let $\left\langle m_{i}\right\rangle$ denote the $K$-submodule of $M$ generated by the $\left\{m_{i}\right\}$ and $M^{\prime \prime}$ denote the $K$-module $M /\left\langle m_{i}\right\rangle$. From the proof of Proposition 1.1 we see that the natural projection of graded $K$-modules $\pi: M \rightarrow M^{\prime \prime}$ induces a map $f: M \rightarrow M^{\prime \prime} \otimes C$ of right $C$-comodules, where $f=(\pi \otimes C) \Delta_{M}$. Because $C$ is flat, the map $f: M \rightarrow M^{\prime \prime} \otimes C$ has a kernel $j: N \rightarrow M$. We can assume $N$ is a subcomodule of $M$ and $j: N \rightarrow M$ is the inclusion. 
1.2. Proposition. In the above situation, $x \in N$ and if $K$ is a Noetherian ring then $N$ is finitely generated as a $K$-module.

Proof. As a graded $K$-module, $N$ is the kernel of $f: M \rightarrow M^{\prime \prime} \otimes C$ in $\mathscr{T} / K$. Hence $N=\left\{m \in M \mid \Delta_{M} m \in\left\langle m_{i}\right\rangle \otimes C\right\}$. It is obvious that $x \in N$. Suppose $y \in$ $M$ and $\Delta_{M} y=\sum m_{r} \otimes c_{r}$ with $m_{r} \in\left\{m_{i}\right\}, c_{r} \in C$. Then $y=\sum m_{r} \varepsilon\left(c_{r}\right)$, so $y \in$ $\left\langle m_{i}\right\rangle$ and $N \subseteq\left\langle m_{i}\right\rangle$. Since $K$ is Noetherian, every submodule of a finitely generated $K$-module is finitely generated. $N$ is therefore a finitely generated $K$-module.

If $C$ is a coalgebra over $K$, let $C^{\text {op }}$ be the coalgebra over $K$ whose underlying graded $K$-module is $C$ and whose comultiplication is $T \circ \Delta_{C}$, where $T$ denotes the twisting morphism. If $M$ is a left $C$-comodule, then $M$ can be given the structure of a right $C^{\text {op }}$-comodule in the obvious manner, namely let the coaction of $C^{\text {op }}$ on $M$ be given by the composition $M \rightarrow C \otimes M \stackrel{T}{\rightarrow} M \otimes C^{\text {op }}$. Similarly, a right $C^{\text {op }}$ comodule is a left $C$-comodule. These constructions facilitate the proof of the following fundamental theorem of coalgebras.

1.3. THEOREM. Let $C$ be a coalgebra over $K$ which is flat as a graded $K$-module and let $x$ be an element of $C$. If $K$ is Noetherian, then there is a finitely generated subcoalgebra of $C$ which contains $x$.

Proof. Suppose $\Delta x=\Sigma c_{(1)} \otimes c_{(2)} . C$ is a right $C \otimes C^{\text {op }}$-comodule by means of the composite

$$
C \stackrel{\Delta}{\rightarrow} C \otimes C \stackrel{C \otimes(T \cdot \Delta)}{\rightarrow} C \otimes\left(C \otimes C^{\mathrm{op}}\right)
$$

By Proposition 1.2, if $i: N \rightarrow C$ is the kernel of the composition

$$
C \rightarrow C \otimes\left(C \otimes C^{\mathrm{op}}\right) \rightarrow C /\left\langle c_{(1)}\right\rangle \otimes\left(C \otimes C^{\mathrm{op}}\right),
$$

then $N$ is a finitely generated right $C \otimes C^{\text {op }}$-comodule containing $x$. But $N$ is then a 2 -sided comodule contained in $C$, so $N$ is a subcoalgebra of $C$.

1.4. Corollary. Suppose $k$ is a field, $C$ is a $k$-coalgebra, and $x$ is an element of $C$. Then there exists a finite-dimensional subcoalgebra of $C$ containing $x$.

The above corollary implies that a coalgebra $C$ over a field $k$ is the union of its finite-dimensional subcoalgebras. Equivalently, such a coalgebra is the direct limit of its finite-dimensional subcoalgebras.

1.5. Definition. A nonzero coalgebra $C$ is said to be simple if it has no nonzero proper subcoalgebras.

1.6. Proposition (CF. [7, LEMMA 8.0.1]). Suppose $C$ is a simple coalgebra over a field $k$ with commutative comultiplication. If $k$ is algebraically closed, then $C$ is one dimensional.

Proof. By Corollary 1.4, $C$ is finite dimensional. Furthermore $C$ is concentrated in degree 0 for otherwise $C_{0}$ would be a nonzero proper subcoalgebra of $C$. $C^{*}$ is then a commutative finite-dimensional $k$-algebra concentrated in degree 0 . As the ideals of $C^{*}$ are in one-to-one correspondence with the subcoalgebras of $C, C^{*}$ is 
simple and is therefore a finite field extension of $k$. Since $k$ is algebraically closed, $\operatorname{dim} C^{*}=\operatorname{dim} C=1$.

1.7. Definition. Let $C$ be a nonzero coalgebra over a field $k$ with commutative comultiplication. If every simple subcoalgebra of $C$ is one dimensional, $C$ is said to be split.

By Proposition 1.6, if $k$ is an algebraically closed field and $C$ is a nonzero commutative $k$-coalgebra, then $C$ is split. The concept of a split coalgebra will play an important role in $\$ 2$.

1.8. Proposition ([7, Proposition 8.0.3]). Let $C$ be a coalgebra over a field $k$ and $\left\{C_{\alpha}\right\}$ a collection of subcoalgebras of $C$ such that $C=\Sigma C_{\alpha}$. Then any simple subcoalgebra of $C$ lies in one of the $C_{\alpha}$.

Proof. Let $D$ be a simple subcoalgebra of $C$. Since $D$ is finite dimensional it lies in some finite sum $\Sigma_{1}^{n} C_{\alpha_{i}}$. By induction on $n$, it suffices to prove that if $D \subseteq C_{\alpha}+$ $C_{\beta}$ then $D \subseteq C_{\alpha}$ or $D \subseteq C_{\beta}$. Suppose $D \nsubseteq C_{\alpha}$. Then since $D$ is simple $D \cap C_{\alpha}=$ 0 . Hence there is $c^{*} \in\left(C_{\alpha}+C_{\beta}\right)^{*}$ such that $c^{*}\left|D=\varepsilon_{D}, c^{*}\right| C_{\alpha}=0$. For $d \in D$,

$$
\sum d_{(1)} c^{*}\left(d_{(2)}\right)=\sum d_{(1)} \varepsilon\left(d_{(2)}\right)=d
$$

where $\Delta_{D} d=\Sigma d_{(1)} \otimes d_{(2)}$. But $D \subseteq C_{\alpha}+C_{\beta}$ implies that

$$
\Delta(D) \subseteq \Delta\left(C_{\alpha}\right)+\Delta\left(C_{\beta}\right) \subseteq C_{\alpha} \otimes C_{\alpha}+C_{\beta} \otimes C_{\beta} .
$$

Since $c^{*} \mid C_{\alpha}=0$, we have $\Sigma d_{(1)} c^{*}\left(d_{(2)}\right) \in C_{\beta}$ and thus $D \subseteq C_{\beta}$.

Now suppose $C$ is a coalgebra over a field $k$. Let $g$ be a set indexing the distinct simple subcoalgebras of $C$. For $j \in \mathcal{G}$, let $C_{j}$ denote the corresponding simple subcoalgebra. $\Sigma_{j \in g} C_{j}$ is then a subcoalgebra of $C$. The previous proposition shows that the sum $\Sigma C_{j}$ is direct. For suppose $C_{s} \cap \sum_{j \neq s} C_{j} \neq 0$ for some $s \in \mathcal{G}$. Then $C_{s} \cap \sum_{j \neq s} C_{j}$ is a nonzero coalgebra contained in $C_{s}$ and hence it must be the case that $C_{s} \cap \Sigma_{j \neq s} C_{j}=C_{s}$. But then $C_{s} \subseteq \Sigma_{j \neq s} C_{j}$, and so $C_{s}=C_{r}$ for some $r \in$ $g-\{s\}$ by Proposition 1.8. This contradicts the fact that $g$ indexes the distinct simple subcoalgebra of $C$. Since the sum $\Sigma C_{j}$ is direct it follows that $\Sigma C_{j} \approx \amalg C_{j}$, where the coproduct of the $C_{j}$ is taken in the category of coalgebras over $k . \amalg_{j \in \mathcal{G}} C_{j}$ is therefore a subcoalgebra of $C$ which we will denote by $S S(C)$, for it is the semi-simple part of $C$. The coalgebra $C$ will be said to be semi-simple if $C=$ $S S(C)$. For future reference we note that a subcoalgebra of a semi-simple coalgebra is semi-simple. For if $D$ is a subcoalgebra of $C=\amalg_{j \in g} C_{j}$ with $C_{j}, j \in \mathscr{g}$, simple, then $D=\amalg_{j \in g}\left(D \cap C_{j}\right)$. In the finite-dimensional case it is easily seen that the ideal $(C / S S(C))^{*}$ in $C^{*}$ is the Jacobson radical of the algebra $C^{*}$, i.e., the intersection of all maximal 2-sided (left, right) ideals. Thus in order to dualize the notion of the Jacobson radical, we consider $C / S S(C)$.

1.9. Defintion. Let $C$ be a coalgebra over a field $k$. Then the coideal $C / S S(C)$ is called the coradical of $C$, and denoted $\operatorname{corad}(C)$.

Recall that Nakayama's lemma states that if $A$ is an algebra, $J(A)$ is the Jacobson radical of $A$, and $Y$ is an $A$-module finitely generated over $A$, then $Y=0$ if and only if $Y \otimes A / J(A)=0$. Nakayama's lemma dualizes to coalgebras and comodules in the following form: 
1.10. Theorem (Dual Nakayama Lemma). Let $C$ be a coalgebra over $a$ field $k$ and let $X$ be a right $C$-comodule. Then $X \square_{C} S S(C)=0$ if and only if $X=0$.

Proof. Clearly we need only prove that $X \square_{c} S S(C)=0$ implies that $X=0$. Let $\pi: C \rightarrow \operatorname{corad}(C)$ be the natural projection. Let $\nu_{n}$ be the composite $C \rightarrow C^{n+1} \stackrel{\pi^{n+1}}{\rightarrow} \operatorname{corad}(C)^{n+1}$, where $C^{n+1}$ denotes the $n+1$-fold tensor product of $C$ with itself and similarly for $\operatorname{corad}(C)^{n+1}$. Denote the kernel of $\nu_{n}$ by $F_{n}(C)$; $F_{0}(C)$ is simply $S S(C) . F_{n}(C)$ is a subcoalgebra of $C$ since $\nu_{n}$ is a mapping of 2-sided $C$-comodules. Furthermore observe that

$$
\begin{aligned}
F_{n}(C)=\left\{x \in C \mid \Delta^{n}(x)\right. & \in S S(C) \otimes C^{n}+C \otimes S S(C) \otimes C^{n-1} \\
& \left.+\cdots+C^{n-1} \otimes S S(C) \otimes C+C^{n} \otimes S S(C)\right\} .
\end{aligned}
$$

Hence by the associativity of comultiplication $F_{n}(C) \subseteq F_{n+1}(C)$.

Assertion 1. $\cup_{n} F_{n}(C)=C$.

Proof. We consider first the finite-dimensional case. Then $\operatorname{corad}(C)^{*}=J\left(C^{*}\right)$, where $J\left(C^{*}\right)$ denotes the Jacobson radical of $C^{*}$. Let $A=C^{*}$. We then have dual diagrams

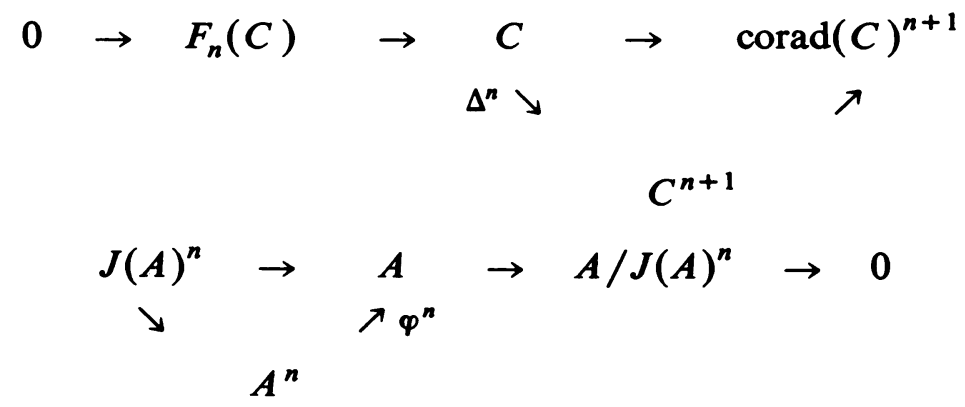

In a finite-dimensional algebra over a field the Jacobson radical is actually nilpotent and hence there exists an integer $N$ such that $J(A)^{N}=0$. In this case $F_{N-1}(C)=C$.

A slight disgression into classical algebra is required at this point. Suppose $R$ and $S$ are rings, and the homomorphism of rings $\tau: R \rightarrow S$ is surjective. Suppose $I \subseteq S$ is a maximal left ideal. Then $\tau^{-1}(I)$ is maximal since $R / \tau^{-1}(I) \approx S / I$ implies that $R / \tau^{-1}(I)$ is a simple left $R$-module. Hence $J(R) \subseteq \cap \tau^{-1}(I)$, where $I$ ranges over all maximal left ideals in $S$, and so $\tau(J(R)) \subseteq J(S)$. There is then an induced morphism $R / J(R)^{n} \rightarrow S / J(S)^{n}$ for $n=0,1,2, \ldots$ Dualizing this, we see that if $C$ and $D$ are finite-dimensional coalgebras and $i: C \rightarrow D$ is injective, then $i\left(F_{n}(C)\right)$ $\subseteq F_{n}(D)$ for $n=0,1,2, \ldots$ Thus if $\left\{C_{\alpha}, i_{\alpha, \beta}\right\}$ is a direct mapping family of coalgebras such that the $i_{\alpha, \beta}$ are injections, then $\lim _{\rightarrow} F_{n}\left(C_{\alpha}\right)=F_{n}\left(\lim C_{\alpha}\right)$. Let $\left\{C_{\alpha}, i_{\alpha, \beta}\right\}$ denote the family of finite-dimensional subcoalgebras of a coalgebra $C$, the $i_{\alpha, \beta}$ being the natural injections. Then $C=\lim _{\rightarrow} C_{\alpha}$ and by the above $F_{n}(C)=\lim _{\rightarrow} F_{n}\left(C_{\alpha}\right)$. But we know that $\cup_{n} F_{n}\left(C_{\alpha}\right)=\vec{C}_{\alpha}$, and hence $\cup_{n} F_{n}(C)=$ C.

Assertion 2. $F_{n+1}(C) / F_{n}(C)$ is a left $S S(C)$-comodule. 
Proof. Again we consider first the finite-dimensional case. In this case we know that $F_{n}(C)$ is dual to $A / J(A)^{n+1}$, where $A=C^{*}$.

$$
\left(F_{n+1}(C) / F_{n}(C)\right)^{*} \approx J(A)^{n+1} / J(A)^{n+2},
$$

which is an $A / J(A)$ module. Thus $F_{n+1}(C) / F_{n}(C)$ is an $(A / J(A))^{*}=S S(C)$ comodule. So for $C_{\alpha}$ finite-dimensional, we have

$$
f_{C_{\alpha}}: F_{n+1}\left(C_{\alpha}\right) / F_{n}\left(C_{\alpha}\right) \rightarrow S S\left(C_{\alpha}\right) \otimes F_{n+1}\left(C_{\alpha}\right) / F_{n}\left(C_{\alpha}\right)
$$

As a coalgebra $C$ is the direct limit of its finite-dimensional subcoalgebras $\left\{C_{\alpha}\right\}$, we have

$$
\lim _{\rightarrow} f_{C_{\alpha}}: \lim _{\rightarrow} F_{n+1}\left(C_{\alpha}\right) / F_{n}\left(C_{\alpha}\right) \rightarrow \lim _{\rightarrow} S S\left(C_{\alpha}\right) \otimes \lim _{\rightarrow} F_{n+1}\left(C_{\alpha}\right) / F_{n}\left(C_{\alpha}\right) .
$$

$\lim _{\rightarrow}$ and $F_{n}$ commute in this case, so the above is simply

$$
\lim _{\rightarrow} f_{C_{a}}: F_{n+1}(C) / F_{n}(C) \rightarrow S S(C) \otimes F_{n+1}(C) / F_{n}(C) .
$$

Hence $F_{n+1}(C) / F_{n}(C)$ is a left $S S(C)$-comodule.

Now the sequence

$$
0 \rightarrow F_{n+1}(C) / F_{n}(C) \rightarrow S S(C) \otimes F_{n+1} / F_{n}(C)
$$

is exact, which implies that

$$
0 \rightarrow X \square_{C} F_{n+1}(C) / F_{n}(C) \rightarrow X \square_{C}\left(S S(C) \otimes F_{n+1}(C) / F_{n}(C)\right)
$$

is exact. $\left(X \square_{C}\right.$ is left exact because we are over a field.) But

$$
X \square_{C}\left(S S(C) \otimes F_{n+1}(C) / F_{n}(C)\right) \approx\left(X \square_{C} S S(C)\right) \otimes F_{n+1}(C) / F_{n}(C)=0,
$$

since $X \square_{c} S S(C)=0$ by assumption. Hence we conclude that $X \square c F_{n+1}(C) / F_{n}(C)=0$. From

$$
0 \rightarrow F_{n}(C) \rightarrow F_{n+1}(C) \rightarrow F_{n+1}(C) / F_{n}(C) \rightarrow 0
$$

we obtain

$$
0 \rightarrow X \square_{C} F_{n}(C) \rightarrow X \square_{C} F_{n+1}(C) \rightarrow X \square_{C} F_{n+1}(C) / F_{n}(C) .
$$

If we assume that $X \square_{C} F_{n}(C)=0$, then the above shows that $X \square_{c} F_{n+1}(C)=0$. Thus by induction $X \square_{C} F_{n}(C)=0$ for all $n>0$, and upon taking direct limits we obtain $X \square_{c} C=X=0$.

Note that in Nakayama's lemma one needs the assumption that the module is finitely generated over the algebra, whereas in the Dual Nakayama Lemma no such finiteness condition is required. This is very characteristic of coalgebras over a field $k$. Because a coalgebra over $k$ is the direct limit of its finite-dimensional subcoalgebras, it is often the case that a property of finite-dimensional $k$-algebras will dualize to a property of arbitrary $k$-coalgebras. Similarly properties of modules finitely generated over a $k$-algebra often dualize to properties of arbitrary comodules over a $k$-coalgebra.

The proof of Theorem 1.10 introduces an important filtration of a coalgebra. As this filtration will be needed later, we establish it now as a definition. 
1.11. Definition. Let $C$ be a coalgebra over a field $k$. For $n=0,1,2, \ldots$, let $F_{n}(C)$ denote the kernel of the composite

$$
C \rightarrow C^{n+1} \stackrel{\pi^{n+1}}{\rightarrow} \operatorname{corad}(C)^{n+1}
$$

This filtration of $C$ is called the coradical filtration.

1.12. Definition. Suppose $C$ is a coalgebra over a ring $K$ with augmentation $\eta$ : $K \rightarrow C$. $C$ is said to be cocomplete if the only simple subcoalgebra of $C$ is $\eta(K)$.

1.13. Corollary. Let $C$ be a supplemented cocomplete coalgebra over a field $k$ and let $f: Y \rightarrow Y^{\prime \prime}$ be a morphism of right $C$-comodules. Then $f$ is injective if and only if $f \square_{c} k: Y \square_{c} k \rightarrow Y^{\prime \prime} \square_{c} k$ is injective.

Proof. If $f$ is injective, then $f \square_{c} k$ is injective since we are over a field. On the other hand, let $i: Y^{\prime} \rightarrow Y$ be the kernel of $f: Y \rightarrow Y^{\prime \prime}$. If $f \square_{c} k$ is injective, then $Y^{\prime} \square_{c} k=0$. But $k \approx S S(C)$ as a left $C$-comodule, and hence $Y^{\prime} \square_{c} k=0$ implies that $Y^{\prime}=0$ by the Dual Nakayama Lemma. It follows that $f$ is injective.

We conclude this section with a basic structure theorem for $k$-coalgebras.

1.14. Definition. A nonzero coalgebra is called local if it contains a unique simple subcoalgebra.

1.15. Proposition (CF. [7, TheOREM 8.0.5]). Suppose $C$ is a coalgebra over a field $k$ with commutative comultiplication and simple subcoalgebras $\left\{C_{j}\right\}_{j \in J}$. Then $C=$ $\amalg_{j \in J} \tilde{C}_{j}$, where $\tilde{C}_{j}$ is a local subcoalgebra of $C$ and $C_{j} \subseteq \tilde{C}_{j} . \tilde{C}_{j}$ is uniquely characterized as being the maximal local subcoalgebra of $C$ containing $C_{j}$.

Proof. First consider the case where $C$ is finite-dimensional. Then $C^{*}$ is a finite-dimensional commutative algebra, and hence is the direct sum of local subalgebras [8, Vol. I, p. 205]. If $C^{*}=A_{1} \oplus \cdots \oplus A_{n}$ with $A_{i}$ a local subalgebra, then $C \approx C^{* *} \approx A_{1}^{*} \oplus \cdots \oplus A_{n}^{*}$ with $A_{i}^{*}$ a local subcoalgebra. Upon observing that this decomposition is unique, the general case follows by taking direct limits. Hence $C$ is a coproduct of local subcoalgebras; suppose $C=\amalg_{\tau \in T} \tilde{C}_{\tau}$. It is clear that each $\tilde{C}_{\tau}$ contains one and only one simple subcoalgebra of $C$. We may therefore reindex the $\left\{\tilde{C}_{\tau}\right\}_{\tau \in T}$ using the index set $J$ such that $\left\{\tilde{C}_{\tau}\right\}_{\tau \in T}=\left\{\tilde{C}_{j}\right\}_{j \in J}$ and $C_{j} \subseteq \tilde{C}_{j}$. If $D$ is a subcoalgebra of $C$ properly containing $\tilde{C}_{i}, i \in J$, then $D \cap \amalg_{i \neq j} \tilde{C}_{j} \neq 0$. Hence there exists a $C_{h}$ with $h \in J, h \neq i$, such that $C_{h} \subseteq D$. D is therefore not a local subcoalgebra and it follows that $\tilde{C}_{i}$ is maximal with respect to the property of being a local subcoalgebra of $C$ containing $C_{i}$. But this uniquely characterizes $\tilde{C}_{i}$; for if $F$ is another local subcoalgebra of $C$ containing $C_{i}$ such that $\tilde{C}_{i} \nsupseteq F$, then $\tilde{C}_{i}+F$ is a local subcoalgebra containiing $C_{i}$ by Proposition 1.8. This violates the maximality of $\tilde{C}_{i}$.

2. Split Hopf algebras with involution. Recall from $\S 1$ that if $C$ is a commutative coalgebra defined over a field $k$, then $C$ is said to be split if every simple subcoalgebra of $C$ is one dimensional. Let $H$ be a Hopf algebra with commutative comultiplication defined over a field $k . H$ is said to be a split Hopf algebra if the underlying coalgebra of $H$ is split. 
2.1. Definition. Let $H$ be a Hopf algebra with commutative comultiplication over a ring $K$. An involution of $H$ is a mapping of coalgebras $c: H \rightarrow H$ such that $\varphi(H \otimes c) \Delta_{H}=\eta \varepsilon$.

If $k$ is a field, we denote by $\mathcal{H} / k$ the category of split Hopf algebras over $k$ with involution.

The significance of the involution becomes apparent from categorical constructions. We proceed as in Milnor and Moore [5, §8]. Let $K$ be a commutative ring, and let $\mathcal{Q}$ denote the category of supplemented coalgebras with commutative comultiplication over $K . \mathbb{Q}$ is a pointed category with products: the point in $\mathscr{Q}$ is $K$ and the product of $A$ and $B$ is just $A \otimes B$. Let $A \times B$ denote the product in $Q$ of $A$ and $B$. A monoid in the category $\mathcal{Q}$ is then an object $C$ together with a morphism $\varphi: C \times C \rightarrow C$ such that the diagrams

$$
\begin{aligned}
& \begin{array}{rlc}
C \times C \times C & \stackrel{\varphi \times C}{\rightarrow} & C \times C \\
\downarrow C \times \varphi & & \downarrow \varphi
\end{array} \\
& C \times C \quad \stackrel{\varphi}{\rightarrow} \quad C \\
& C=K \times C \stackrel{\eta \times C}{\rightarrow} \quad C \times C \\
& C=C \times \underbrace{\stackrel{C \times \eta}{\rightarrow}}_{C} C \times C
\end{aligned}
$$

are commutative. Thus a monoid in the category $\mathbb{Q}$ is simply a Hopf algebra with commutative comultiplication. A group in the category is a monoid in the category together with $h: C \rightarrow C$ such that the diagram

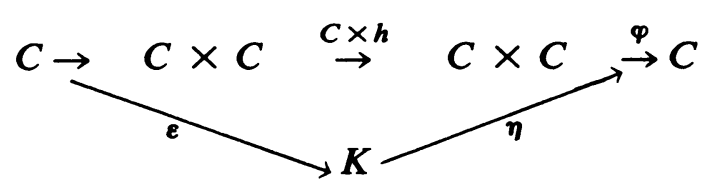

is commutative. Hopf algebras with commutative comultiplication and involution are groups in the category $\mathcal{Q}$. Similarly $\mathcal{K} / k$ is the category of groups in the category of supplemented split $k$-coalgebras. In light of the above comments, it is not surprising that much of our work has its motivation in the theory of groups.

If $H$ is a Hopf algebra over a field $k$, we can view $H$ as a coalgebra and consider $S S(H)$.

2.2. Proposition. If $H \in$ obj $\mathcal{X} / k$, then $S S(H)$ is a sub-Hopf algebra of $H$. 
Proof. $k \eta(1)$ is a simple subcoalgebra of $H$, so $k \eta(1) \subseteq S S(H)$ and thus $\eta(1) \in S S(H)$. Since all simple subcoalgebras of $H$ are one dimensional, $S S(H)$ is the coproduct of cofields. Suppose $S S(H)=\amalg_{j \in J} k e_{j}^{\prime}$, with $k e_{j}^{\prime}$ a simple subcoalgebra. Then $\Delta e_{j}^{\prime}=r_{j} e_{j}^{\prime} \otimes e_{j}^{\prime}$ for some $0 \neq r_{j} \in k$. Letting $e_{j}=r_{j} e_{j}^{\prime}$, we see that $\Delta e_{j}=e_{j} \otimes e_{j}$. With $e_{j}$ chosen as the basis element for $k e_{j}^{\prime}$ we can write $S S(H)=$ $\amalg_{j \in J} k e_{j}, \Delta e_{j}=e_{j} \otimes e_{j}$. For $i, j \in J$,

$$
\Delta \varphi\left(e_{i} \otimes e_{j}\right)=\varphi\left(e_{i} \otimes e_{j}\right) \otimes \varphi\left(e_{i} \otimes e_{j}\right)
$$

and hence $k \varphi\left(e_{i} \otimes e_{j}\right)$ is a simple subcoalgebra of $H$. Then $k \varphi\left(e_{i} \otimes e_{j}\right) \subseteq S S(H)$ and $S S(H)$ is closed under multiplication.

Note that if $G$ is a group, the group algebra $k G$ is easily made into a Hopf algebra by defining $\Delta g=g \otimes g$ for all $g \in G$. By abuse of language, we shall refer to the Hopf algebra $k G$ as the group algebra on the group $G$.

2.3. Definition. Suppose $H$ is a Hopf algebra over a commutative ring $K$. Define $G(H)$ to be $\{x \in H \mid \Delta x=x \otimes x$ and $x \neq 0\}$.

\subsection{Proposition. If $H \in \mathrm{obj} \mathcal{H} / k$, then $S S(H)$ is a group algebra.}

Proof. We will show that $G(H)$ is a group with multiplication $\varphi_{H} \mid G(H)$. By the proof of Proposition 2.2, $G(H)$ is closed under multiplication. $\eta(1)$ is the identity in $G(H)$. Suppose $x \in G(H)$. Then $(H \otimes \varepsilon) \Delta x=\varepsilon(x) x=x$, and hence $\varepsilon(x)=1$. Let $c: H \rightarrow H$ be the involution. By the definition of $c, \varphi(H \otimes c) \Delta x=\eta \varepsilon(x)=$ $\eta(1)$. Thus $x$ is $\varphi$-invertible with multiplicative inverse $c(x)$. Since $\Delta x=x \otimes x$ and $c$ is a morphism of coalgebras, $\Delta c(x)=c(x) \otimes c(x)$ and so $c(x) \in G(H)$. It is now trivial to see that $S S(H)$ is a group algebra on the group $G(H)$.

2.5. Definition. Let $H$ be a Hopf algebra over a commutative ring $K$. For notational convenience, identify $K$ and $K \eta(1) \subseteq H$. Let $\zeta_{n}, n=0,1,2, \ldots$, denote the composition

$$
H \stackrel{\Delta^{n}}{\rightarrow} H^{n+1} \stackrel{\pi^{n+1}}{\rightarrow}(H / K)^{n+1} .
$$

Denote by $\mathscr{F}_{n}(H)$ the kernel of $\zeta_{n}$. Since $\zeta_{n}$ is a mapping of 2-sided $H$-comodules, $\mathscr{F}_{n}(H)$ is a subcoalgebra of $H$. Moreover $\mathscr{F}_{n}(H) \subseteq \mathscr{F}_{n+1}(H), n=0,1,2, \cdots$. Let $C(H)=\cup_{n} \mathscr{F}_{n}(H) . C(H)$ is the cocomplete part of $H$. (Note that if $H \in$ obj $\mathcal{H} / k$, then $C(H)$ is cocomplete in the sense of Definition 1.12.) If $H=C(H)$, $H$ is said to be a cocomplete Hopf algebra.

2.6. Proposition. If $H \in$ obj $\mathcal{K} / k$, then $C(H)$ is a sub-Hopf algebra of $H$.

Proof. Since the $\left\{\mathscr{F}_{n}(H)\right\}$ are an increasing tower of subcoalgebras, $C(H)$ is a subcoalgebra. $\eta(1) \in \mathscr{F}_{0}(H)$, so $C(H)$ contains the multiplicative identity. We need only show that $C(H)$ is closed under multiplication. So suppose $\alpha \in \mathscr{F}_{n}(H)$, $\beta \in \mathscr{F}_{m}(H)$. Then

$$
\begin{gathered}
\Delta^{n} \alpha \in k \otimes H^{n}+H \otimes k \otimes H^{n-1}+\cdots+H^{n} \otimes k, \\
\Delta^{m} \beta \in k \otimes H^{m}+H \otimes k \otimes H^{m-1}+\cdots+H^{m} \otimes k .
\end{gathered}
$$

Each term in $\Delta^{n+m} \alpha$ will contain $m+1$ elements of $k$ whereas each term in $\Delta^{n+m} \beta$ will contain $n+1$ elements of $k$. By the definition of a Hopf algebra, 


$$
\Delta \varphi(\alpha \otimes \beta)=\sum(-1)^{\operatorname{deg} \alpha(2) \operatorname{deg} \beta_{(1)}} \varphi\left(\alpha_{(1)} \otimes \beta_{(1)}\right) \otimes \varphi\left(\alpha_{(2)} \otimes \beta_{(2)}\right)
$$

if $\Delta \alpha=\sum \alpha_{(1)} \otimes \alpha_{(2)}, \Delta \beta=\sum \beta_{(1)} \otimes \beta_{(2)}$. By induction it follows that

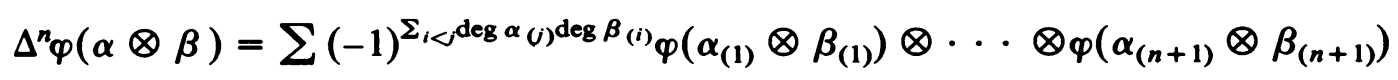

if

$$
\Delta^{n} \alpha=\sum \alpha_{(1)} \otimes \cdots \otimes \alpha_{(n+1)}, \quad \Delta^{n} \beta=\sum \beta_{(1)} \otimes \cdots \otimes \beta_{(n+1)} .
$$

Hence when $\Delta^{n+m} \varphi(\alpha \otimes \beta)$ is computed, the expressions for $\Delta^{n+m} \alpha$ and $\Delta^{n+m} \beta$ are multiplied component-wise. Since each term in $\Delta^{n+m} \alpha$ contains $m+1$ elements of $k$ and each term in $\Delta^{n+m} \beta$ contains $n+1$ elements of $k$, there exists at least one component in each term where both $\Delta^{n+m} \alpha$ and $\Delta^{n+m} \beta$ have an element of $k$. Thus $\varphi(\alpha \otimes \beta) \in \mathscr{F}_{n+m}(H)$.

2.7. Comments. Let $H \in$ obj $\mathcal{H} / k$. Observe that $G(H)$ acts on $S S(H)$ by conjugation: if $h \in S S(H)$ and $g \in G(H)$, then $g h g^{-1} \in S S(H)$. SS $(H)$ is a normal sub-Hopf algebra of $H$ (the precise meaning of this will be given in $\$ 5$ ), and $G(H)$ is the quotient of $H$ by $S S(H)$ as Hopf algebras.

2.8. THEOREM. If $H \in \mathrm{obj} \mathcal{H} / k$, then $H \approx \amalg_{g \in G(H)} C(H) g$ as a coalgebra.

Proof. $C(H)$ is clearly a local subcoalgebra since by definition any cocomplete coalgebra is local. Suppose $L$ is a local subcoalgebra of $H$ such that $1 \in L$. Let $F_{n}(L)$ denote the coradical filtration on $L . F_{n}(L) \subseteq C(H)$ for $n=0,1,2, \ldots$ and so $L \subseteq C(H)$ since $\cup F_{n}(L)=L$. Hence $C(H)$ is the maximal local subcoalgebra of $H$ containing 1. Let $H^{(g)}$ denote the maximal local subcoalgebra of $H$ containing $g \in G(H)$. If $h \in H^{(g)}$ then for some $n, h \in F_{n}\left(H^{(g)}\right)$, implying that $h g^{-1} \in$ $F_{n}(C(H))=\mathscr{F}_{n}(H)$. Since $h g^{-1} \in C(H), h \in C(H) g$ and hence $H^{(g)} \subseteq C(H) g$. But visibly $C(H) g \subseteq H^{(g)}$, and it follows that $C(H) g=H^{(g)}$. By Proposition 1.15, $H \approx \amalg_{g \in G(H)} C(G) g$.

Let $K$ be a commutative ring. Recall that if $A$ is a supplemented $K$-algebra with augmentation ideal $I(A)$, then the elements of $Q(A)=K \otimes_{A} I(A)$ are called the indecomposable elements of $A$. If $C$ is a supplemented $K$-coalgebra and $C=K \oplus$ $J(C)$ as a graded $K$-module, then the elements of $P(C)=K \square_{C} J(C)$ are called the primitive elements of $C$. The primitive elements of $C$ can be naturally identified with $\{x \in C \mid \Delta x=x \otimes 1+1 \otimes x\}$. If $H$ is a Hopf algebra, there is a natural morphism of graded $K$-modules $P(H) \rightarrow Q(H)$. Also the primitive elements of $H$ form a Lie algebra with Lie product

$$
[x, y]=x y-(-1)^{p q} y x \quad \text { for } x \in H_{p}, y \in H_{q} .
$$

2.9. Definition AND Comments [5, pp. 237, 238]. If $L, L^{\prime}$ are Lie algebras over $K$, then $L \times L^{\prime}$ is the Lie algebra over $K$ such that

$$
\left[(x, y),\left(x^{\prime}, y^{\prime}\right)\right]=\left(\left[x, x^{\prime}\right],\left[y, y^{\prime}\right]\right)
$$

for

$$
(x, y) \in\left(L \times L^{\prime}\right)_{p}=L_{p} \times L_{p}^{\prime}, \quad\left(x^{\prime}, y^{\prime}\right) \in\left(L \times L^{\prime}\right)_{q}=L_{q} \times L_{q}^{\prime}
$$


Now if $L$ is a Lie algebra there is a natural morphism $\Delta: L \rightarrow L \times L$, where $\Delta(x)=(x, x)$ for $x \in L_{n}$. This induces

$$
U(\Delta): U(L) \rightarrow U(L) \otimes U(L)=U(L \times L)
$$

and $U(L)$ becomes a Hopf algebra. Henceforth we will consider the universal enveloping algebra of a Lie algebra to be a Hopf algebra with the above comultiplication.

2.10. ThEOREM. Suppose $k$ is a field of characteristic 0 and $H$ is a Hopf algera over $k$. Then $C(H)$ is the universal enveloping algebra of the Lie algebra $P(H)=$ $P(C(H))$.

Proof. We have a natural map $m: U(P(C(H))) \rightarrow C(H)$ which sends $x_{1}$ $\otimes \cdots \otimes x_{n}$ to $x_{1} \cdots x_{n}$, where $x_{i} \in P(C(H)), 1<i<n$. Filter $U(P(C(H)))$ by the Lie filtration, i.e. let $F_{p}(U(P(C(H))))$ be the $k$-submodule generated by elements of length $<p$. Filter $C(H)$ by the coradical filtration.

Assertion. The map $m: U(P(C(H))) \rightarrow C(H)$ is filtration preserving.

Proof. The proof proceeds by induction on $p$, starting from $p=0$. For $p=0$ we have $F_{0}(C(H))=k, F_{0}(U(P(C(H))))=k ; m: F_{0}(U(P(C(H)))) \rightarrow F_{0}(C(H))$ is just the identity. Suppose

$$
m\left(F_{p-1}(U(P(C(H))))\right) \subseteq F_{p-1}(C(H))
$$

for some $p>1$. Let $x_{1} \otimes \cdots \otimes x_{p}$ be an element of $F_{p}(U(P(C(H))))$, where $x_{1}, \ldots, x_{p} \in P(C(H))$. Then $\Delta^{p}\left(x_{1} \cdots x_{p}\right)$ can be calculated by multiplying $\Delta^{p}\left(x_{1} \cdots x_{p-1}\right)$ and $\Delta^{p}\left(x_{p}\right)$ component-wise. By assumption $x_{1} \cdots x_{p-1} \in$ $F_{p-1}(C(H))$, so $\Delta^{p-1}\left(x_{1} \cdots x_{p-1}\right)$ has at least one $k$-entry in each term. Hence $\Delta^{p}\left(x_{1} \cdots x_{p-1}\right)$ has at least two $k$-entries in each term. Since $\Delta x_{p}=x_{p} \otimes 1+1 \otimes$ $x_{p}$,

$\Delta^{p} x_{p}=x_{p} \otimes 1 \otimes \cdots \otimes 1+1 \otimes x_{p} \otimes 1 \otimes \cdots \otimes 1+\cdots+1 \otimes \cdots \otimes 1 \otimes x_{p}$.

Multiplying component-wise we see that $\Delta^{p}\left(x_{1} \cdots x_{p}\right)$ must have at least one entry from $k$ in each term. Hence $x_{1} \cdots x_{p} \in F_{p}(C(H))$.

Since the map $m$ is filtration preserving, we may consider

$$
E^{\circ}(U(P(C(H)))) \stackrel{E^{\circ}(m)}{\rightarrow} E^{\circ}(C(H)) .
$$

$E^{\circ}(U(P(C(H))))$ and $E^{\circ}(C(H))$ are bigraded Hopf algebras; the induced morphism $E^{\circ}(m)$ is a morphism of bigraded Hopf algebras. One sees easily that $E^{\circ}(C(H))$ and $E^{\circ}(U(P(C(H))))$ are connected: $E^{\circ}(C(H))_{0,0}=k$ and $E^{\circ}(C(H))_{0, q}=0$ for $q \neq 0$, similarly $E^{\circ}(U(P(C(H))))_{0,0}=k$ and $E^{\circ}(U(P(C(H))))_{0, q}=0$ for $q \neq 0$.

We now draw heavily on results established in [5].

Since $\operatorname{char}(k)=0$, the natural morphism of graded $K$-modules

$$
P\left(E^{\circ}(U(P(C(H))))\right) \rightarrow Q\left(E^{\circ}(U(P(C(H))))\right)
$$

is an isomorphism [5, Proposition 5.13]. Also $P\left(E^{\circ}(U(P(C(H))))\right)_{p, q}=0$ for $p \neq 1$, $P\left(E^{\circ}(U(P(C(H))))\right)_{1, q}=P(U(P(C(H))))_{q+1}=P(C(H))_{q+1}$ [5, Propositions 5.11 and 5.13, Theorem 5.18]. Observe that 


$$
\begin{aligned}
& P\left(E^{\circ}(C(H))\right)_{p, q}=0 \text { for } p \neq 1, \\
& P\left(E^{\circ}(C(H))\right)_{1, q}=P(C(H))_{q+1} ;
\end{aligned}
$$

these statements are immediate consequences of the appropriate definitions. The mapping $E^{\circ}(m)$ is the identity on $P(C(H))$, and therefore $E^{\circ}(m)$ injects [5, Proposition 3.9]. To show that $E^{\circ}(m)$ surjects, it suffices to show that

$$
Q\left(E^{\circ}(U(P(C(H))))\right) \rightarrow Q\left(E^{\circ}(C(H))\right)
$$

surjects [5, Proposition 3.8]. However, this follows from the diagram

$$
\begin{array}{ccc}
P\left(E^{\circ}(U(P(C(H))))\right) & \stackrel{\approx}{\rightarrow} & P\left(E^{\circ}(C(H))\right) \\
\downarrow \approx & & \downarrow \\
Q\left(E^{\circ}(U(P(C(H))))\right) & \rightarrow & Q\left(E^{\circ}(C(H))\right)
\end{array}
$$

$P\left(E^{\circ}(C(H))\right) \rightarrow Q\left(E^{\circ}(C(H))\right)$ surjects because the comultiplication in $H$ is commutative and $\operatorname{char}(k)=0$ [5, Proposition 4.18]. Because $E^{\circ}(m)$ is an isomorphism, $m$ is an isomorphism and the theorem is established.

2.11. Corollary. Suppose $k$ is a field of characteristic 0 and $H \in$ obj $\mathcal{X} / k$. Then as coalgebras $H \approx \amalg_{g \in G(H)} U(P(H)) g$.

3. Analogues of Lagrange's theorem for Hopf algebras. If $H$ is a Hopf algebra and $H^{\prime} \subseteq H$ is a sub-Hopf algebra, then $H$ is in a natural way an $H^{\prime}$-module. When is $H$ a free $H^{\prime}$-module such that 1 is a possible basis element? Note that this question simply asks to what extent Lagrange's theorem generalizes to Hopf algebras. In this section we shall see that $H$ is always a free $H^{\prime}$-module with 1 a possible basis element if $H, H^{\prime} \in$ obj $\mathcal{H} / k$.

However, it is also true that the above generalization of Lagrange's theorem is valid under certain conditions if $\boldsymbol{H}$ and $\boldsymbol{H}^{\prime}$ are universal enveloping algebras of free Lie algebras. Although this fact is irrelevant to the main course of our argument, we prove it here because it gives a significant analogue of a group theoretical result for a class of Hopf algebras whose ground ring is not assumed to be a field.

Suppose $K$ is a commutative ring with $2 \in K$ a unit. Let $L$ be a graded Lie algebra over $K$ which is a free $K$-module in each degree.

3.1. Definitions and Notation. Suppose $L_{i}$ is free on the set $\left\{x_{i, \theta}\right\}_{(i, \theta) \in \Theta_{i}}$. Choose a simple ordering for the disjoint union $\cup_{i \text { odd }} \Theta_{i}$, and a simple ordering for the disjoint union $\cup_{i \text { even }} \Theta_{i}$. Obtain a simple ordering for $\cup_{i} \Theta_{i}$ by setting $(i, \theta)<(j, \lambda)$ whenever $i$ is even and $j$ is odd. For $x_{j, \theta} \in L$, denote by $y_{j, \theta}$ the element $i\left(x_{j, \theta}\right)$ of $U(L)$, where $i: L \rightarrow U(L)$ is the natural injection. If $I=$ $\left(i_{1}, \theta_{1}\right), \ldots,\left(i_{n}, \theta_{n}\right)$ is a sequence of indices such that $i_{1}, \ldots, i_{m}(0<m<n)$ are even; $i_{m+1}, \ldots, i_{n}$ are odd,

$$
\left(i_{1}, \theta_{1}\right) \leqslant\left(i_{2}, \theta_{2}\right)<\cdots<\left(i_{m}, \theta_{m}\right)
$$

and

$$
\left(i_{m+1}, \theta_{m+1}\right)<\left(i_{m+2}, \theta_{m+2}\right)<\cdots<\left(i_{n}, \theta_{n}\right),
$$

call the sequence $I$ semi-strictly increasing. If $I=\left(i_{1}, \theta_{1}\right), \ldots,\left(i_{n}, \theta_{n}\right)$ is a finite sequence of indices, its length is $n$ and its degree is $i_{1}+\cdots+i_{n}, y_{i_{1}, \theta_{1}} \cdots y_{i_{n}, \theta_{n}}$ 
shall be denoted by $y_{I}$. The empty set is assumed to be semi-strictly increasing with $y_{J}=1$ if $J=\varnothing$.

3.2. Theorem (Graded Poincare-Birkhoff-Witt) (CF. [1, Chapter 13]). With L as above, $U(L)_{n}$ is free on the set $\left\{y_{I}\right\}$, where $I$ ranges over all finite semi-strictly increasing sequences of degree $n$.

Proof. We first show that the elements $y_{I}$ corresponding to finite semi-strictly increasing sequences of degree $n$ generate $U(L)_{n}$. Let $F_{p}\left(U(L)_{n}\right)$ denote those elements of $U(L)_{n}$ of length $<p$. It clearly suffices to show that the elements $\left\{y_{I}\right\}$, where $I$ ranges over all finite semi-strictly increasing sequences of length $<p$ and degree $n$, generate $F_{p}\left(U(L)_{n}\right)$. This follows by recursion from the following lemma:

3.3. LemmA. For each sequence $a_{1}, \ldots, a_{p} \in L$ such that $i\left(a_{1}\right) i\left(a_{2}\right) \cdots i\left(a_{p}\right) \in$ $U(L)_{n}$ and each permutation $\pi$ of $(1,2, \ldots, p)$ we have

$$
i\left(a_{1}\right) \cdots i\left(a_{p}\right)+i\left(a_{\pi(1)}\right) \cdots i\left(a_{\pi(p)}\right) \in F_{p-1}\left(U(L)_{n}\right) \text {. }
$$

or

$$
i\left(a_{1}\right) \cdots i\left(a_{p}\right)-i\left(a_{\pi(1)}\right) \cdots i\left(a_{\pi(p)}\right) \in F_{p-1}\left(U(L)_{n}\right) .
$$

Proof. It is enough to consider the case where $\pi$ interchanges two consecutive indices $j, j+1$. In this case the statement is evident from the relations:

$$
i\left(a_{j}\right) i\left(a_{j+1}\right)+i\left(a_{j+1}\right) i\left(a_{j}\right)=i\left(\left[a_{j}, a_{j+1}\right]\right),
$$

$a_{j} \in L_{u}, a_{j+1} \in L_{v}, u, v$ odd

otherwise.

$$
i\left(a_{j}\right) i\left(a_{j+1}\right)-i\left(a_{j+1}\right) i\left(a_{j}\right)=i\left(\left[a_{j}, a_{j+1}\right]\right)
$$

Now recall that a left representation of the Lie algebra $L$ over $K$ is a $K$-module $M$ together with a $K$-homomorphism $x \otimes m \rightarrow x m$ of $L \otimes M$ into $M$ such that

$$
x(y m)-(-1)^{p q} y(x m)=[x, y] m \text { for } x \in L_{p}, y \in L_{q}, m \in M .
$$

Let $A$ be the algebra formed by tensoring the symmetric algebra on the even degrees of $L$ with the exterior algebra on the odd degrees of $L$. Denote the generator of $A$ corresponding to $x_{i, \theta} \in L_{i}$ by $z_{i, \theta}$. If $I=\left(i_{1}, \theta_{1}\right), \ldots,\left(i_{n}, \theta_{n}\right)$ is a finite sequence of indices and $(j, \tau) \in \Theta_{j}$, say that $(j, \tau)<I$ if $(j, \tau)<\left(i_{s}, \theta_{s}\right)$ for $s=1,2, \ldots, n$. Similarly say that $(j, \tau)<I$ if $(j, \tau)<\left(i_{s}, \theta_{s}\right)$ for $s=1,2, \ldots, n$.

3.4. Lemma. There exists a left representation of $L$ in $A$ such that

$$
x_{i, \theta} z_{I}=z_{i, \theta} z_{I}
$$

for $(i, \theta)<I, i$ even, or $(i, \theta)<I, i$ odd.

Assuming Lemma 3.4 we can complete the proof of the theorem. The representation of $L$ in $A$ induces a left $U(L)$-module structure on $A$. If $I$ is a semi-strictly increasing sequence of indices of length $m$ it follows from (1) by recursion on $m$ that $y_{I} \cdot 1=z_{I}$. As the elements $z_{I}$ are $K$-linearly independent in $A$, the elements $y_{I}$ must be $K$-linearly independent in $U(L)$. Hence $U(L)_{n}$ is a free $K$-module with basis $\left\{y_{I}\right\}$, where $I$ ranges over all finite semi-strictly increasing sequences of degree $n$. 
Proof of 3.4. Filter $A$ by length; let $Q_{p}$ denote those elements of length $<p$. The proof follows from the following inductive assertion:

$\left(S_{p}\right)$ For each integer $p \geqslant 0$ there is a unique homomorphism $f: L \otimes Q_{p} \rightarrow A$ such that

$$
f\left(x_{i, \theta} \otimes z_{I}\right)=z_{i, \theta} z_{I}
$$

$i$ even, $(i, \theta)<I, z_{I} \in Q_{p}$, or $i$ odd, $(i, \theta)<I, z_{I} \in Q_{p}$,

$$
\begin{gathered}
f\left(x_{i, \theta} \otimes z_{I}\right) \in Q_{q+1}, \quad z_{I} \in Q_{q}, q<p, \\
f\left(x_{i, \theta} \otimes f\left(x_{j, \tau} \otimes z_{J}\right)\right)=(-1)^{i j} f\left(x_{j, \tau} \otimes f\left(x_{i, \theta} \otimes z_{J}\right)\right) \\
+f\left(\left[x_{i, \theta}, x_{j, \tau}\right] \otimes z_{J}\right), \quad z_{J} \in Q_{p-1}, \\
f\left(x_{i, \theta} \otimes z_{I}\right)-z_{i, \theta} z_{I} \in Q_{q}, \quad z_{I} \in Q_{q}, q<p .
\end{gathered}
$$

For $p=0$, the definition $f\left(x_{i, \theta} \otimes 1\right)=z_{i, \theta}$ is forced by $\left(1^{\prime}\right)$ and satisfies (2)-(4). Assume that $\left(S_{p-1}\right)$ has been established for some $p>0$. We must define $f\left(x_{i, \theta} \otimes\right.$ $\left.z_{I}\right)$ for $I$ of length $p$. If $(i, \theta) \leqslant I, i$ even, or $(i, \theta)<I, i$ odd, then by $\left(1^{\prime}\right)$ we must define $f\left(x_{i, \theta} \otimes z_{I}\right)=z_{i, \theta} z_{I}$. If the above is false then $I$ may be uniquely written as $I=((j, \tau), J)$ where

$$
\begin{array}{ll}
(i, \theta)>(j, \tau)<J, & i \text { even, } \\
(i, \theta)>(j, \tau)<J, & i \text { odd, } j \text { even, } \\
(i, \theta) \geqslant(j, \tau)<J, & i \text { odd, } j \text { odd. }
\end{array}
$$

Then $z_{I}=z_{j, \tau} z_{J}=f\left(x_{j, \tau} \otimes z_{J}\right)$, so the left side of (3) is $f\left(x_{i, \theta} \otimes z_{I}\right)$. We must show that the right side of (3) is already defined. Use (4) to write

$$
f\left(x_{i, \theta} \otimes z_{J}\right)=z_{i, \theta} z_{J}+w, \quad w \in Q_{p-1} .
$$

The right side of (3) becomes

$$
\begin{aligned}
(-1)^{i j} f\left(x_{j, \tau} \otimes\left(z_{i, \theta} z_{J}+w\right)\right)+ & f\left(\left[x_{i, \theta}, x_{j, \tau}\right] \otimes z_{J}\right) \\
& =(-1)^{i j} z_{j, \tau} z_{i, \theta} z_{J}+(-1)^{i j} f\left(x_{j, \tau} \otimes w\right)+f\left(\left[x_{i, \tau}, x_{j, \tau}\right] \otimes z_{J}\right)
\end{aligned}
$$

if it is not the case that $i, j$ odd, $(i, \theta)=(j, \tau)<J$. If $i$ and $j$ are odd, $(i, \theta)=(j, \tau)$ $<J$, let

$$
f\left(x_{i, \theta} \otimes z_{I}\right)=\frac{1}{2} f\left(\left[x_{i, \theta}, x_{i, \theta}\right] \otimes z_{J}\right) .
$$

This defines $f$ in all cases, and (1), (2), (4) of $\left(S_{p}\right)$ are satisfied. (3) is verified for the case $(i, \theta)>(j, \tau)<J, j$ even, or $(i, \theta) \geqslant(j, \tau)<J, j$ odd. Because of the antisymmetry of $\left[x_{i, \theta}, x_{j, \tau}\right],(3)$ is also true for $(j, \tau) \geqslant(i, \theta)<J, i$ even, or $(j, \tau)>$ $(i, \theta)<J, i$ odd. Suppose $(j, \tau)=(i, \theta)$ and $J=((i, \theta), L)$ with $i, j$ odd. Then it can be seen without difficulty that

$$
\begin{aligned}
f\left(x_{i, \theta} \otimes f\left(x_{i, \theta} \otimes z_{J}\right)\right)= & -f\left(x_{i, \theta} \otimes f\left(x_{i, \theta} \otimes z_{J}\right)\right) \\
& +f\left(\left[x_{i, \theta}, x_{i, \theta}\right] \otimes z_{J}\right)+f\left(\left[x_{i, \theta}\left[x_{i, \theta}, x_{i, \theta}\right]\right] \otimes z_{J}\right) .
\end{aligned}
$$

But $\left[x_{i, \theta}\left[x_{i, \theta}, x_{i, \theta}\right]\right]=0$, so (3) is satisfied for the case $(i, \theta)>(j, \tau)\langle J$ or $(j, \tau)\rangle$ $(i, \theta)<J$. In order to minimize notational confusion, henceforth we simply use lower case Greek letters for subscripts, denoting by the Greek letter both the subscript and the dimension of the element. 
Suppose neither $\theta<J$ nor $\tau \leqslant J$. Then $J=(\nu, L)$ where $\nu<L$, $\nu$ even, or $\nu<L$, $\nu$ odd; $\nu<\theta, \nu<\tau$. Then either $\nu$ is even or all of $J, \theta, \tau$ are odd by virtue of the way the simple ordering on the indices was defined.

Note that

$$
\begin{aligned}
x_{\tau} z_{J} & =x_{\tau}\left(x_{\nu} z_{L}\right)=(-1)^{\tau \nu} x_{\nu}\left(x_{\tau} z_{L}\right)+\left[x_{\tau}, x_{\nu}\right] z_{L} \\
& =(-1)^{\tau \nu} x_{\nu}\left(z_{\tau} z_{L}\right)+(-1)^{\tau \nu} x_{\nu} w+\left[x_{\tau}, x_{\nu}\right] z_{L},
\end{aligned}
$$

where $w=x_{\tau} z_{L}-z_{\tau} z_{L} \in Q_{p-2}$. Applying $x_{\theta}$ to both sides we obtain after algebraic manipulation

$$
\begin{aligned}
x_{\theta}\left(x_{\tau} z_{J}\right)= & (-1)^{(\theta+\tau) \nu} x_{\nu}\left(x_{\theta}\left(x_{\tau} z_{L}\right)\right)+(-1)^{\tau \nu}\left[x_{\theta}, x_{\nu}\right]\left(x_{\tau} z_{L}\right) \\
& +(-1)^{(\tau+\nu) \theta}\left[x_{\tau}, x_{\nu}\right]\left(x_{\theta} z_{L}\right)+\left[x_{\theta},\left[x_{\tau}, x_{\nu}\right]\right] z_{L} .
\end{aligned}
$$

Similarly,

$$
\begin{aligned}
x_{\tau}\left(x_{\theta} z_{J}\right)= & (-1)^{(\tau+\theta) \nu} x_{\nu}\left(x_{\tau}\left(x_{\theta} z_{L}\right)\right)+(-1)^{\theta \nu}\left[x_{\tau}, x_{\nu}\right]\left(x_{\theta} z_{L}\right) \\
& +(-1)^{(\theta+\nu) \tau}\left[x_{\theta}, x_{\nu}\right]\left(x_{\tau} z_{L}\right)+\left[x_{\tau},\left[x_{\theta}, x_{\nu}\right]\right] z_{L} .
\end{aligned}
$$

If $\nu$ is even, then

$$
\begin{aligned}
& x_{\theta}\left(x_{\tau} z_{J}\right)-(-1)^{\theta \tau} x_{\tau}\left(x_{\theta} z_{J}\right)= x_{\nu}\left\{x_{\theta}\left(x_{\tau} z_{L}\right)-(-1)^{\theta \tau} x_{\tau}\left(x_{\theta} z_{L}\right)\right\} \\
&+\left[x_{\theta},\left[x_{\tau}, x_{\nu}\right]\right] z_{L}-(-1)^{\theta \tau}\left[x_{\tau},\left[x_{\theta}, x_{\nu}\right]\right] z_{L} . \\
& x_{\nu}\left\{x_{\theta}\left(x_{\tau} z_{L}\right)-(-1)^{\theta \tau} x_{\tau}\left(x_{\theta} z_{L}\right)\right\}=\left[x_{\theta}, x_{\tau}\right] z_{J}+\left[x_{\nu},\left[x_{\theta}, x_{\tau}\right]\right] z_{L}, \\
& {\left[x_{\theta},\left[x_{\tau}, x_{\nu}\right]\right]-(-1)^{\theta \tau}\left[x_{\tau},\left[x_{\theta}, x_{\nu}\right]\right]+\left[x_{\nu},\left[x_{\theta}, x_{\tau}\right]\right]=0 }
\end{aligned}
$$

by Jacobi's identity, and therefore

$$
x_{\theta}\left(x_{\tau} z_{J}\right)-(-1)^{\theta \tau} x_{\tau}\left(x_{\theta} z_{J}\right)=\left[x_{\theta}, x_{\tau}\right] z_{J} .
$$

The case where $\nu, \theta, \tau$ are odd is treated similarly.

3.5. CoRollary. Let $K$ be a commutative ring such that 2 is a unit in $K$, and let $L$ be a free Lie algebra over $K$. Filter $U(L)$ by the Lie filtration as defined in the proof of Theorem 3.2, namely let $F_{p}\left(U(L)_{n}\right)$ denote those elements of $U(L)_{n}$ of length $<p$. If $L_{i}$ is free on $\left\{x_{i, \theta}\right\}_{(i, \theta) \in \Theta_{i}}$, then $E^{\circ}(U(L))$ is isomorphic to a free commutative algebra whose ith dimension has generators $\left\{x_{i, \theta}\right\}_{(i, \theta) \in \theta_{i}}$.

3.6. Corollary. Let $L$ be a free Lie algebra over $K$, where $K$ is as above. Suppose $L^{\prime}$ is a sub-Lie algebra of $L$ such that a basis for $L^{\prime}$ can be extended to a basis for $L$. Then $U(L)$ regarded as a left (or right) $U\left(L^{\prime}\right)$-module is free with 1 a possible basis element.

Proof. Suppose $L_{i}^{\prime}$ is free on the set $\left\{x_{i, \theta}\right\}_{(i, \theta) \in \theta_{i}}$, and $L_{i}$ is free on the set $\left\{x_{i, \theta}, y_{i, \tau}\right\}_{(i, \theta) \in \theta_{i},(i, \tau) \in T_{i}}$. Choose a simple ordering for the disjoint union $\bigcup_{i}\left\{x_{i, \theta}, y_{i, \tau}\right\}$ as in the proof of Theorem 3.2. Let $M$ denote the graded $K$-module such that $M_{n}$ is free on all finite semi-strictly increasing sequences $y_{i_{1}, \tau_{1}} \cdots y_{h_{1}, \tau_{n}}$ of degree $n$. Consider the natural morphism of $U\left(L^{\prime}\right)$-modules $U\left(L^{\prime}\right) \otimes M \rightarrow U(L)$ defined by $x_{i_{1}, \theta_{1}} \cdots x_{i_{m}, \theta_{m}} \otimes y_{i_{1}, \tau_{n}} \cdots y_{i_{n}, \tau_{n}} \mapsto x_{i_{1}, \theta_{1}} \cdots x_{i_{m}, \theta_{m}} y_{i_{1}, \tau_{1}} \cdots y_{i_{n}, \tau_{n}}$, where 
we allow ourselves the obvious notational abuse of denoting an element of a Lie algebra and its image in the universal enveloping algebra by the same symbol. We wish to show that this morphism $U\left(L^{\prime}\right) \otimes M \rightarrow U(L)$ is an isomorphism of left $U\left(L^{\prime}\right)$-modules. Filtering $M$ by length it is immediate that the mapping $U\left(L^{\prime}\right) \otimes M$ $\rightarrow U(L)$ is filtration preserving, and passing to the associated bigraded modules we have $E^{\circ}\left(U\left(L^{\prime}\right)\right) \otimes E^{\circ}(M) \rightarrow E^{\circ}(U(L))$. This is obviously an isomorphism and hence $U\left(L^{\prime}\right) \otimes M \approx U(L)$.

We now turn our attention to split Hopf algebras with involution.

3.7. Theorem. Suppose $H \in$ obj $\mathcal{K} / k$, and $H^{\prime} \in$ obj $\mathcal{K} / k$ is a sub-Hopf algebra of $H$. Then $H$ is a free left (or right) $H^{\prime}$-module, and one can choose a basis for $H$ over $H^{\prime}$ which includes 1 .

Proof. The proof of this theorem is divided into several parts. We first prove the theorem for group algebras and cocomplete Hopf algebras, and then show how the general result follows from this.

Assertion 1. Let $G$ be a group and consider the group algebra $k G \in$ obj $\mathcal{H} / k$. Suppose $H^{\prime} \in$ obj $\mathcal{H} / k$ is a sub-Hopf algebra of $k G$. Then $k G$ is a free left (or right) $H^{\prime}$-module, and a basis can be chosen for $k G$ over $H$ which includes 1 .

Proof. Since $H^{\prime}$ is a subcoalgebra of the semi-simple coalgebra $k G, H^{\prime}$ must be semi-simple as a coalgebra. (See the discussion following the proof of Proposition 1.8.) It follows by Proposition 2.4 that $H^{\prime}$ is a group algebra because $H \in$ obj $\mathcal{H} / k$. We therefore have $H^{\prime}=k L$, where $L$ is a subgroup of $G$, and the result is evident by Lagrange's Theorem for groups.

Assertion 2. Let $H$ be a cocomplete Hopf algebra over the field $k$. If $H^{\prime}$ is a sub-Hopf algebra of $H$, then $H$ is a free left (or right) $H^{\prime}$-module with 1 being a possible basis element.

Proof. Let $M=k \otimes_{H^{\prime}} H$. We have the natural projection $H \rightarrow M$. Filter $M$ by defining $F_{p}(M)=\operatorname{Im} F_{p}(H)$, where $H$ is given the coradical filtration. Inductively define a section $\rho: M \rightarrow H$ such that $\rho$ is filtration preserving. Then the composition

$$
h: H^{\prime} \otimes\left(k \otimes_{H^{\prime}} H\right) \stackrel{i \otimes \rho}{\rightarrow} H \otimes H \stackrel{\varphi_{H}}{\rightarrow} H
$$

is filtration preserving, where $H^{\prime}$ is filtered by the coradical filtration. Passing to the $E^{\circ}$ level we obtain

$$
\begin{gathered}
E^{\circ}(h): E^{\circ}\left(H^{\prime}\right) \otimes\left(k \otimes_{E^{\circ}\left(H^{\prime}\right)} E^{\circ}(H)\right) \stackrel{E^{\circ}(i) \otimes E^{\circ}(\rho)}{\rightarrow} E^{\circ}(H) \otimes E^{\circ}(H) \\
\stackrel{E^{\circ}\left(\varphi_{H}\right)}{\rightarrow} E^{\circ}(H),
\end{gathered}
$$

since $E^{\circ}(M) \approx k \otimes_{E^{\circ}\left(H^{\prime}\right)} E^{\circ}(H)$. This is the bigraded connected case, and here we know that $E^{\circ}(h)$ is an isomorphism of left $E^{\circ}\left(H^{\prime}\right)$-modules [5, Proposition 1.7]. But this implies that $h$ is an isomorphism of left $H^{\prime}$-modules and the assertion is established.

We now suppose $H, H^{\prime} \in$ obj $\mathcal{H} / k$ such that $H^{\prime} \subseteq H$ and $G\left(H^{\prime}\right)=G(H)$. Then $k \otimes_{H^{\prime}} H \approx k \otimes_{C\left(H^{\prime}\right)} C(H)$. Let $\rho: k \otimes_{C\left(H^{\prime}\right)} C(H) \rightarrow C(H)$ be a filtration preserving section where $C(H)$ and $C\left(H^{\prime}\right)$ are given the coradical filtration and 
$k \otimes_{C\left(H^{\prime}\right)} C(H) \approx k \otimes_{H^{\prime}} H$ is filtered as in the proof of Assertion 2 above. The composition

$$
r: H^{\prime} \otimes\left(k \otimes_{C\left(H^{\prime}\right)} C(H)\right) \stackrel{i \otimes_{\rho}}{\rightarrow} H \otimes H \stackrel{\varphi_{H}}{\rightarrow} H
$$

is a morphism of left $H^{\prime}$-modules. Furthermore

$$
r \mid C\left(H^{\prime}\right) \otimes\left(k \otimes_{C\left(H^{\prime}\right)} C(H)\right): C\left(H^{\prime}\right) \otimes\left(k \otimes_{C\left(H^{\prime}\right)} C(H)\right) \rightarrow C(H)
$$

is an isomorphism of left $C\left(H^{\prime}\right)$-modules by Assertion 2. Since $H^{\prime} \approx$ $\amalg_{g \in G\left(H^{\prime}\right)} g C\left(H^{\prime}\right)$ as a coalgebra, any element $0 \neq h^{\prime}$ of $H^{\prime}$ can be written as $\sum_{j=1}^{n} g_{j} h_{j}^{\prime}$, where $0 \neq h_{j}^{\prime} \in C\left(H^{\prime}\right)$ for $1 \leqslant j \leqslant n$ and $g_{j} \in G\left(H^{\prime}\right)$ with $g_{i} \neq g_{j}$ if $i \neq j, 1<i, j<n$. Let $0 \neq \alpha \in k \otimes_{C(H)} C(H)$. Then $r\left(h^{\prime} \otimes \alpha\right)=\sum_{j=1}^{n} g_{j} r\left(h_{j}^{\prime} \otimes \alpha\right)$. $r\left(h_{j}^{\prime} \otimes \alpha\right)$ is a nonzero element of $C(H)$ since $r \mid C\left(H^{\prime}\right) \otimes\left(k \otimes_{C\left(H^{\prime}\right)} C(H)\right)$ is bijective. Because $H \approx \amalg_{g \in G(H)} g C(H), \sum_{j=1}^{n} g_{j} r\left(h_{j}^{\prime} \otimes \alpha\right)$ cannot equal 0 . Since $h^{\prime} \otimes \alpha$ was an arbitrary nonzero element of $H^{\prime} \otimes\left(k \otimes_{C(H)} C(H)\right)$ it follows that $r$ injects.

To show that $r$ surjects it suffices to show that all elements of the form $g \tilde{h}$, $g \in G, \tilde{h} \in C(H)$ are hit by $r$. Let $\sum_{i=1}^{n} \tilde{h}_{i}^{\prime} \otimes \alpha_{i} \in C\left(H^{\prime}\right) \otimes\left(k \otimes_{C\left(H^{\prime}\right)} C(H)\right)$ such that $r\left(\sum_{i=1}^{n} \tilde{h_{i}^{\prime}} \otimes \alpha_{i}\right)=\tilde{h}$. Then

$$
r\left(\sum_{i=1}^{n} g \tilde{h_{i}^{\prime}} \otimes \alpha_{i}\right)=g \tilde{h}
$$

Hence

$$
H^{\prime} \otimes\left(k \otimes_{H^{\prime}} H\right) \approx H^{\prime} \otimes\left(k \otimes_{C\left(H^{\prime}\right)} C(H)\right) \stackrel{i \otimes \rho}{\rightarrow} H \otimes H \stackrel{\varphi_{H}}{\rightarrow} H
$$

is an isomorphism of left $H^{\prime}$-modules if $G\left(H^{\prime}\right)=G(H)$. Similarly one shows that if $H, H^{\prime} \in$ obj $\mathcal{H} / k$ such that $H^{\prime} \subseteq H$ and $C(H)=C\left(H^{\prime}\right)$, then

$$
H^{\prime} \otimes\left(k \otimes_{H^{\prime}} H\right) \stackrel{i \otimes \tau}{\rightarrow} H \otimes H \stackrel{\varphi_{H}}{\rightarrow} H
$$

is an isomorphism of left $H^{\prime}$-modules. In this case $\tau: k \otimes_{H^{\prime}} H \approx k \otimes_{k G\left(H^{\prime}\right)} k G(H)$ $\rightarrow H$ is the section induced by choosing a set of coset representatives for $G\left(H^{\prime}\right)$ in $G(H)$ which includes the identity.

For the general case suppose $H^{\prime}, H \in$ obj $\mathcal{K} / k$ and $H^{\prime} \subseteq H$. Let $H^{\prime \prime}$ be the subcoalgebra of $H$ isomorphic to $k G\left(H^{\prime}\right) \otimes C(H)$. For $g^{\prime} \in G\left(H^{\prime}\right), h \in C(H)$, we know that $g^{\prime} h g^{\prime-1} \in C(H)$. Hence $H^{\prime \prime}$ is a sub-Hopf algebra of $H$ with $G\left(H^{\prime \prime}\right)=$ $G\left(H^{\prime}\right)$ and $C\left(H^{\prime \prime}\right)=C(H)$. Furthermore $H^{\prime \prime}$ has an involution. We then have isomorphisms:

$$
\begin{gathered}
H^{\prime} \otimes\left(k \otimes_{H^{\prime}} H^{\prime \prime}\right) \stackrel{i \otimes \rho}{\rightarrow} H^{\prime \prime} \otimes H^{\prime \prime} \stackrel{\varphi_{H^{\prime}}}{\rightarrow} H^{\prime \prime}, \\
H^{\prime \prime} \otimes\left(k \otimes_{H^{\prime \prime}} H\right) \stackrel{i \otimes \tau}{\rightarrow} H \otimes H \stackrel{\varphi_{H}}{\rightarrow} H .
\end{gathered}
$$

It follows that $H^{\prime} \otimes\left(k \otimes_{H^{\prime}} H\right) \otimes\left(k \otimes_{H^{\prime \prime}} H\right) \approx H^{\prime} \otimes\left(k \otimes_{H^{\prime}} H\right) \approx H$ as left $H^{\prime}$-modules.

4. Epimorphisms in $\mathcal{K} / k$. Let $\mathscr{D}$ be a category with point *. Suppose $D^{\prime}, D^{\prime \prime} \in$ obj $\mathscr{D}$ and $f: D^{\prime} \rightarrow D^{\prime \prime}$ is an epimorphism. The epimorphism $f: D^{\prime} \rightarrow D^{\prime \prime}$ is said to be normal if $f$ has a kernel $\xi: N \rightarrow D^{\prime}$ and $f$ is the cokernel of $\xi$. Dually a monomorphism $g: D^{\prime} \rightarrow D^{\prime \prime}$ is said to be normal if $g$ has a cokernel $\zeta: D^{\prime \prime} \rightarrow M$, 
and $g$ is the kernel of $\zeta$. It is the aim of this section to show that every epimorphism in $\mathcal{K} / k$ is a normal epimorphism. Several technical results concerning algebras must be established first, and we proceed following Lemaire [4].

4.1. Definition. Let $i: A \rightarrow B$ and $j: A \rightarrow C$ be two monomorphisms in a category $C$. Then the amalgamated coproduct of $B$ and $C$ with respect to $A$, denoted $B \amalg_{A} C$, is the pushout object of the diagram

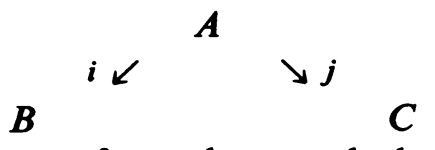

Let $\mathscr{Q} \lg / K$ denote the category of supplemented algebras over the commutative ring $K$. If $A \in$ obj $Q \lg / K$, denote by $\bar{A}$ the augmentation ideal of $A$. For $B, C \in$ obj $\mathscr{Q} \lg / K$,

$$
B \amalg C=k \oplus(\bar{B} \oplus \bar{C}) \oplus(\bar{B} \otimes \bar{C} \oplus \bar{C} \otimes \bar{B}) \oplus \cdots
$$

Also given monomorphisms $i: A \rightarrow B, j: A \rightarrow C$ in $\mathcal{Q} \lg / K, B \amalg_{A} C=B \amalg C / I$, where $I$ denotes the ideal in $B \amalg C$ generated by $\{i(a)-j(a) \mid a \in A\}$.

4.2. Definition. Let

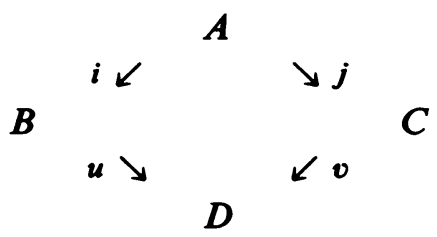

be a cocartesian square in $Q \lg / K$ with $i, j$ injections. This diagram will be said to satisfy the condition $[H]$ in case there exist sections

$$
\begin{aligned}
& \psi: K \otimes_{A} B \rightarrow B \\
& \tau: K \otimes_{A} C \rightarrow C
\end{aligned} \quad \text { with } \psi(\overline{1})=1, \tau(\overline{1})=1,
$$

such that

$$
\begin{aligned}
& \tilde{\psi}: A \otimes\left(K \otimes_{A} B\right) \stackrel{i \otimes \psi}{\rightarrow} B \otimes B \stackrel{\varphi_{B}}{\rightarrow} B, \\
& \tilde{\tau}: A \otimes\left(K \otimes_{A} C\right) \stackrel{j \otimes \tau}{\rightarrow} C \otimes C \stackrel{\varphi_{C}}{\rightarrow} C
\end{aligned}
$$

are isomorphisms of left $A$-modules.

4.3. Notation. We adopt the following notation with regard to the diagram

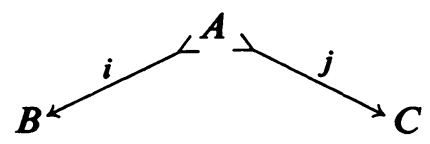

in $\mathcal{Q} \lg / K$ :

$$
\begin{array}{rlrl}
X & =K \otimes_{A} B, & \bar{X} & =\operatorname{Ker}\left(\varepsilon: K \otimes_{A} B \rightarrow K\right), \\
Y=K \otimes_{A} C, & \bar{Y}=\operatorname{Ker}\left(\varepsilon: K \otimes_{A} C \rightarrow K\right) .
\end{array}
$$

For $p>0$,

$$
\begin{array}{ll}
\bar{X}^{1}=\bar{X}, & \bar{Y}^{1}=\bar{Y} \\
\bar{X}^{2 p}=\bar{X}^{2 p-1} \otimes \bar{Y}, & \bar{Y}^{2 p}=\bar{Y}^{2 p-1} \otimes \bar{X}, \\
\bar{X}^{2 p+1}=\bar{X}^{2 p} \otimes \bar{X}, & \bar{Y}^{2 p+1}=\bar{Y}^{2 p} \otimes \bar{Y} .
\end{array}
$$


Now set

$$
\begin{aligned}
& R=K \oplus\left(\bigoplus_{p} \bar{X}^{p}\right) \oplus\left(\bigoplus_{p} \bar{Y}^{p}\right), \\
& S=K \oplus\left(\bigoplus_{p} \bar{Y}^{p}\right), \\
& T=K \oplus\left(\bigoplus_{P} \bar{X}^{p}\right) .
\end{aligned}
$$

4.4. Proposition. Let

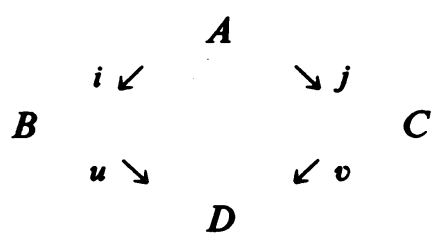

be a cocartesian square in $\mathbb{Q} \lg / K$ with $i, j$ injections. If $(1)$ satisfies the condition $[H]$, then (1) is a cartesian square in $\mathbb{Q l g} / K$.

Proof. Let $\psi: X \rightarrow B$ and $\tau: Y \rightarrow C$ be sections such that $\tilde{\psi}: A \otimes X \rightarrow B$ and $\tilde{\tau}$ : $A \otimes Y \rightarrow C$ are isomorphisms of left $A$-modules. From the definitions it is apparent that $R=X \otimes S=Y \otimes T$, and hence we have the following isomorphisms of left $A$-modules:

$$
\begin{aligned}
& A \otimes R=A \otimes X \otimes S \underset{\tilde{\tau} \otimes S}{\stackrel{\approx}{\rightleftarrows}} B \otimes S, \\
& A \otimes R=A \otimes Y \otimes T \stackrel{\tilde{\tau} \otimes T}{\rightleftarrows} C \otimes T .
\end{aligned}
$$

These isomorphisms give to $A \otimes R$ left $B$ - and $C$-module structures such that the left $A$-module structures induced via $i$ and $j$ are the same as the initial $A$-module structure. This is equivalent to having the commutative diagram

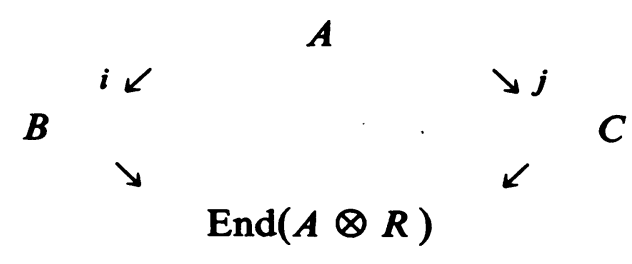

in $\mathscr{Q} \lg / K$. Since $D$ is the pushout object, there is a unique morphism $\eta: D \rightarrow$ End $(A \otimes R)$ such that the diagram

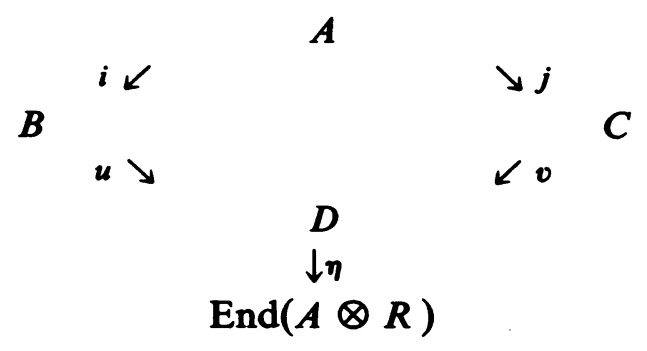


is commutative. Hence a $D$-module structure on $A \otimes R$ is induced. Let $\alpha: D \rightarrow A$ $\otimes R$ by $\alpha(d)=d(1 \otimes 1)$. Then the following diagram commutes:

$A$

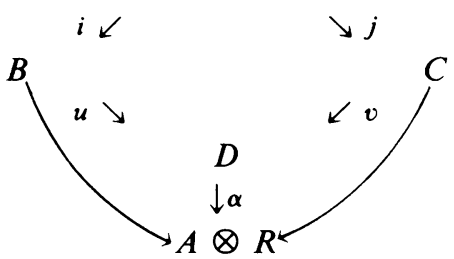

Since $B \rightarrow A \otimes R$ and $C \rightarrow A \otimes R$ are injections, $u$ and $v$ are injections. Visibly the intersection of $B$ and $C$ in $A \otimes R$ is $A$; it then follows that the intersection of $B$ and $C$ in $D$ is $A$. Hence the square (1) is cartesian.

4.5. Corollary. Suppose $f: A \rightarrow B$ is an epimorphism in $\mathbb{Q} \lg / K$. Let $i: f(A) \rightarrow B$ denote the natural inclusion. If the diagram

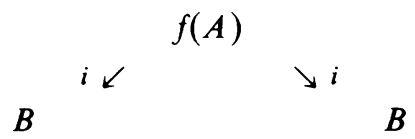

satisfies the condition $[H]$, then $f$ is surjective.

Proof. We consider the pushout of the above diagram

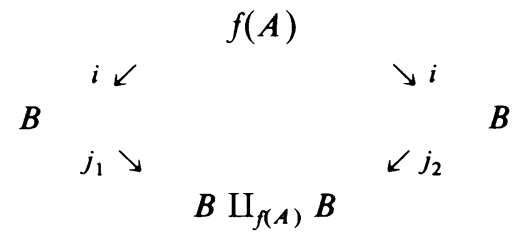

By Proposition 4.4 the square (2) is cartesian, and hence

$$
\left\{b \in B \mid j_{1}(b)=j_{2}(b)\right\}=i f(A) .
$$

However $i: f(A) \rightarrow B$ is epimorphic because $f: A \rightarrow B$ is epimorphic. Thus $j_{1} i=j_{2} i$ implies that $j_{1}=j_{2}$, and so $\left\{b \in B \mid j_{1}(b)=j_{2}(b)\right\}=B$. It follows that if $(A)=B$, and $f$ is surjective.

Now let $f: H \rightarrow H^{\prime \prime}$ be an epimorphism in $\mathcal{K} / k$, and let $i: f(H) \rightarrow H^{\prime \prime}$ denote the inclusion. The diagram

$$
\begin{array}{ll}
i \swarrow & f(H) \\
H^{\prime \prime} & \searrow i \\
& H^{\prime \prime}
\end{array}
$$

satisfies the condition $[H]$ by the results of $\S 3$. Corollary 4.5 then implies that $f(H)=H^{\prime \prime}$.

4.6. THEOREM. Every epimorphism in $\mathcal{H} / k$ is surjective.

Utilizing the fact that epimorphisms are surjective in $\mathcal{H} / k$, we shall prove that every epimorphism in $\mathcal{H} / k$ is normal. The proof is divided into several parts. First we establish the result for group algebras and cocomplete Hopf algebras; then we 
derive the general case from this via the structure theorems for objects in $\mathcal{H} / k$ proved in $\$ 2$.

4.7. Proposition. Suppose $k G, k H$ are group algebras with $f: k H \rightarrow k G$ an epimorphism. Then $f$ is normal.

Proof. $f=f \mid H: H \rightarrow G$ is an epimorphism in $\mathcal{G}$, the category of groups. Let $\tilde{i}$ : $N \rightarrow H$ be the kernel of $f: H \rightarrow G$ in $\mathcal{G}$. Then there is induced a morphism of group algebras $i: k N \rightarrow k H$. One verifies without difficulty that $f \circ i=*, i: k N \rightarrow k H$ is the kernel of $f: k H \rightarrow k G$, and $f: k H \rightarrow k G$ is the cokernel of $i: k N \rightarrow k H$.

4.8. Proposition. Suppose $H, H^{\prime \prime} \in$ obj $\mathcal{H} / k$ are cocomplete Hopf algebras and $g: H \rightarrow H^{\prime \prime}$ is an epimorphism. Then $g$ is normal.

Proof. Let $H^{\prime}$ denote $k \square_{H^{\prime \prime}} H$ and $i: H^{\prime} \rightarrow H$ denote the natural injection. By definition $k \square_{H^{\prime \prime}} H$ is the graded $k$-module such that the sequence

$$
0 \rightarrow k \square_{H^{\prime \prime}} H \rightarrow k \otimes H^{\Delta_{k} \otimes H-k \otimes \Delta_{H}} \stackrel{\rightarrow}{\rightarrow} \otimes H^{\prime \prime} \otimes H
$$

is exact. Here $\Delta_{k}$ refers to the composition

$$
k \rightarrow k \otimes k \stackrel{k \otimes \eta}{\rightarrow} k \otimes H^{\prime \prime}
$$

and $\Delta_{H}$ refers to the composition

$$
H \rightarrow H \otimes H^{g} \stackrel{\otimes H}{\rightarrow} H^{\prime \prime} \otimes H .
$$

However it is easily seen that $k \square_{H^{\prime \prime}} H$ can be given the structure of a $k$-algebra such that $i: k \square_{H^{\prime \prime}} H \rightarrow H$ is a morphism of algebras [5, Proposition 4.6]. Furthermore it is possible to show that $k \square_{H^{\prime \prime}} H$ can be given the structure of a coalgebra such that $i: k \square_{H^{\prime \prime}} H \rightarrow H$ is a morphism of $k$-coalgebras. This is due to the fact that $H$ and $H^{\prime \prime}$ have commutative comultiplication and the ground ring $k$ is a field [5, Proposition 3.6]. Hence $H^{\prime}=k \square_{H^{\prime \prime}} H$ is actually a Hopf algebra with $i$ : $H^{\prime} \rightarrow H$ a morphism of Hopf algebras.

Assertion. $H \approx H^{\prime \prime} \otimes H^{\prime}$ as left $H^{\prime \prime}$-comodules and right $H^{\prime}$-modules.

Proof. Filter $H$ by the coradical filtration; filter $H^{\prime \prime}$ by $F_{n}\left(H^{\prime \prime}\right)=\operatorname{Im} F_{n}(H)$. This filtration on $H^{\prime \prime}$ is complete since $g$ is surjective by Theorem 4.5. Choose a mapping of graded $k$-modules $r: H \rightarrow H^{\prime}$ such that $r$ is filtration preserving and $r \circ i=H^{\prime}$. Let $\tilde{g}$ be the composite of the mappings

$$
H \stackrel{\Delta_{H}}{\rightarrow} H \otimes H^{g} \stackrel{\otimes r}{\rightarrow} H^{\prime \prime} \otimes H^{\prime} .
$$

Then $\tilde{g}$ is a morphism of left $H^{\prime \prime}$-comodules; $\tilde{g}$ is filtration preserving where $H^{\prime \prime} \otimes H^{\prime}$ is given the tensor product filtration with respect to the filtrations on $H^{\prime \prime}$ and $H^{\prime}$ already defined. Passing to the associated bigraded modules, we have

$$
E^{\circ}(\tilde{g}): E^{\circ}(H) \rightarrow E^{\circ}\left(H^{\prime \prime}\right) \otimes E^{\circ}\left(H^{\prime}\right) .
$$

This then induces

$$
k \square_{E^{\circ}\left(H^{\prime \prime}\right)} E^{\circ}(H) \rightarrow k \square_{E^{\circ}\left(H^{\prime \prime}\right)}\left(E^{\circ}\left(H^{\prime \prime}\right) \otimes E^{\circ}\left(H^{\prime}\right)\right) \approx E^{\circ}\left(H^{\prime}\right) .
$$

$k \square_{H^{\prime \prime}} \tilde{\boldsymbol{g}}: \boldsymbol{H}^{\prime} \rightarrow H^{\prime}$ is the identity, so

$$
E^{\circ}\left(k \square_{H^{\prime \prime}} \tilde{\boldsymbol{g}}\right)=k \square_{E^{\circ}\left(H^{\prime \prime}\right)} E^{\circ}(\tilde{g}): E^{\circ}\left(H^{\prime}\right) \rightarrow E^{\circ}\left(H^{\prime}\right)
$$


is the identity. Since $k \square_{E^{\circ}\left(H^{\prime \prime}\right)} E^{\circ}(\tilde{g})$ is a monomorphism, $E^{\circ}(\tilde{g})$ is a monomorphism by Corollary 1.13. This then implies that $\tilde{g}$ is a monomorphism.

We now wish to show that $\tilde{g}$ is an epimorphism. Define $\varphi: H \otimes H^{\prime} \rightarrow H$ by $\varphi\left(h \otimes h^{\prime}\right)=\varphi_{H}\left(h \otimes i\left(h^{\prime}\right)\right)$ for $h \in H, h^{\prime} \in H^{\prime}$. Consider the sequence

$$
H \otimes H^{\prime} \stackrel{\varphi}{\rightarrow} H \stackrel{\Delta_{H}}{\rightarrow} H \otimes H^{g} \stackrel{\otimes_{r}}{\rightarrow} H^{\prime \prime} \otimes H^{\prime} .
$$

All maps are filtration preserving, so there is an induced sequence

$$
\begin{gathered}
E^{\circ}(H) \otimes E^{\circ}\left(H^{\prime}\right) \stackrel{E^{\circ}(\varphi)}{\rightarrow} E^{\circ}(H) \stackrel{E^{\circ}\left(\Delta_{H}\right)}{\rightarrow} E^{\circ}(H) \otimes E^{\circ}(H) \\
\stackrel{E^{\circ}(g) \otimes E^{\circ}(r)}{\rightarrow} E^{\circ}\left(H^{\prime \prime}\right) \otimes E^{\circ}\left(H^{\prime}\right) .
\end{gathered}
$$

Define filtrations on $E^{\circ}(H) \otimes E^{\circ}\left(H^{\prime}\right)$ and $E^{\circ}\left(H^{\prime \prime}\right) \otimes E^{\circ}\left(H^{\prime}\right)$ as follows:

$$
\begin{gathered}
F^{p, q}\left(E^{\circ}\left(H^{\prime \prime}\right) \otimes E^{\circ}\left(H^{\prime}\right)\right)=\sum_{r>p, s>q} E^{\circ}\left(H^{\prime \prime}\right) \otimes E^{\circ}\left(H^{\prime}\right)_{r, s}, \\
F^{p, q}\left(E^{\circ}(H) \otimes E^{\circ}\left(H^{\prime}\right)\right)=\sum_{r>p, s>q} E^{\circ}(H) \otimes E^{\circ}\left(H^{\prime}\right)_{r, s \cdot} .
\end{gathered}
$$

Recall that if $A=\left\{A_{p, q}\right\}$ is a bigraded module and $F$ is a filtration on $A$, then

$$
E^{\circ}(A)_{u, v, r, s}=\left(F_{u, v}(A) / F_{u-1, v-1}(A)\right)_{u+r, v+s^{\circ}}
$$

With the above filtrations on $E^{\circ}\left(H^{\prime \prime}\right) \otimes E^{\circ}\left(H^{\prime}\right)$ and $E^{\circ}(H) \otimes E^{\circ}\left(H^{\prime}\right)$, let $E_{\circ}\left(E^{\circ}\left(H^{\prime \prime}\right) \otimes E^{\circ}\left(H^{\prime}\right)\right)$ and $E_{\circ}\left(E^{\circ}(H) \otimes E^{\circ}\left(H^{\prime}\right)\right)$ denote the associated 4graded modules.

$$
E_{\circ}^{p, q, r, s}\left(E^{\circ}\left(H^{\prime \prime}\right) \otimes E^{\circ}\left(H^{\prime}\right)\right)=E^{\circ}\left(H^{\prime \prime}\right)_{r, s} \otimes E^{\circ}\left(H^{\prime}\right)_{p, q}
$$

and

$$
E_{\circ}^{p, q, r, s}\left(E^{\circ}(H) \otimes E^{\circ}\left(H^{\prime}\right)\right)=E^{\circ}(H)_{r, s} \otimes E^{\circ}\left(H^{\prime}\right)_{p, q} .
$$

Utilizing the commutative diagram

$$
\begin{array}{ccc}
E^{\circ}(H) \otimes E^{\circ}\left(H^{\prime}\right) & \stackrel{E^{\circ}(\varphi)}{\rightarrow} & E^{\circ}(H) \\
E^{\circ}(\Delta) \otimes E^{\circ}\left(H^{\prime}\right) \downarrow & & \downarrow E^{\circ}(\Delta) \\
E^{\circ}\left(H^{\prime \prime}\right) \otimes E^{\circ}(H) \otimes E^{\circ}\left(H^{\prime}\right) & E^{\circ}\left(H^{\prime \prime}\right) \otimes E^{\circ}(\varphi) & E^{\circ}\left(H^{\prime \prime}\right) \otimes E^{\circ}(H)
\end{array}
$$

it is easily seen that

$$
E^{\circ}(\tilde{g} \varphi)\left(F^{p, q}\left(E^{\circ}(H) \otimes E^{\circ}\left(H^{\prime}\right)\right) \subseteq F^{p, q}\left(E^{\circ}\left(H^{\prime \prime}\right) \otimes E^{\circ}\left(H^{\prime}\right)\right) .\right.
$$

Hence

$$
E_{\circ}\left(E^{\circ}(\tilde{g} \varphi)\right): E_{\circ}\left(E^{\circ}(H) \otimes E^{\circ}\left(H^{\prime}\right)\right) \rightarrow E_{\circ}\left(E^{\circ}\left(H^{\prime \prime}\right) \otimes E^{\circ}\left(H^{\prime}\right)\right)
$$

is induced. Identifying $E_{\circ}\left(E^{\circ}(H) \otimes E^{\circ}\left(H^{\prime}\right)\right)$ with $E^{\circ}(H) \otimes E^{\circ}\left(H^{\prime}\right)$ and $E_{\circ}\left(E^{\circ}\left(H^{\prime \prime}\right) \otimes E^{\circ}\left(H^{\prime}\right)\right)$ with $E^{\circ}\left(H^{\prime \prime}\right) \otimes E^{\circ}\left(H^{\prime}\right)$, we have $E_{\circ}\left(E^{\circ}(\tilde{g} \varphi)\right)=E^{\circ}(g)$ $\otimes E^{\circ}\left(H^{\prime}\right)$ which is epimorphic. This implies that $\tilde{g} \varphi$ is epic, which in turn implies that $\tilde{g}$ is epic. Hence $\tilde{g}$ is an isomorphism of left $H^{\prime \prime}$-comodules.

Because $\tilde{\boldsymbol{g}}$ is an isomorphism of left $\boldsymbol{H}^{\prime \prime}$-comodules, there exists a morphism of left $H^{\prime \prime}$-comodules $w: H^{\prime \prime} \rightarrow H$ such that $g w=H^{\prime \prime}$. (Namely $w$ is the composition $H^{\prime \prime} \rightarrow H^{\prime \prime} \otimes H^{\tilde{g}^{-1}} \rightarrow H$.) Let $h$ be the composition: 


$$
H^{\prime \prime} \otimes H^{\prime} \stackrel{w \otimes i}{\rightarrow} H \otimes H \stackrel{\varphi_{H}}{\rightarrow} H .
$$

$h$ is a morphism of left $H^{\prime \prime}$-comodules and right $H^{\prime}$-modules. Consider

$$
\tilde{g} \circ h: H^{\prime \prime} \otimes H^{\prime} \stackrel{w \otimes i}{\rightarrow} H \otimes H \stackrel{\varphi_{H}}{\rightarrow} H \stackrel{\Delta_{H}}{\rightarrow} H \otimes H^{g \otimes r} \rightarrow H^{\prime \prime} \otimes H^{\prime} .
$$

All maps are filtration preserving, so there is induced

$$
E^{\circ}(\tilde{g} h): E^{\circ}\left(H^{\prime \prime}\right) \otimes E^{\circ}\left(H^{\prime}\right) \rightarrow E^{\circ}\left(H^{\prime \prime}\right) \otimes E^{\circ}\left(H^{\prime}\right) .
$$

Filter $E^{\circ}\left(H^{\prime \prime}\right) \otimes E^{\circ}\left(H^{\prime}\right)$ by

$$
F^{p, q}\left(E^{\circ}\left(H^{\prime \prime}\right) \otimes E^{\circ}\left(H^{\prime}\right)\right)=\sum_{r>p, s>q} E^{\circ}\left(H^{\prime \prime}\right) \otimes E^{\circ}\left(H^{\prime}\right)_{r, s \cdot}
$$

Then $E^{\circ}(\tilde{g} h)$ is filtration preserving and $E_{\circ}\left(E^{\circ}(\tilde{g} h)\right)$ is just the identity morphism of $E_{\circ}\left(E^{\circ}\left(H^{\prime \prime}\right)\right) \otimes E_{\circ}\left(E^{\circ}\left(H^{\prime}\right)\right)$. Since $E_{\circ}\left(E^{\circ}(g h)\right)$ is an isomorphism, $\tilde{g} h$ is an isomorphism. This then implies that $h$ is an isomorphism.

It is now a simple matter to show that Proposition 4.7 follows from the above assertion. One sees immediately that $i: H^{\prime} \rightarrow H$ is the kernel of $g: H \rightarrow H^{\prime \prime}$. By the assertion above there is an isomorphism of left $H^{\prime \prime}$-comodules and right $H^{\prime}$-modules $h: H^{\prime \prime} \otimes H^{\prime} \rightarrow H$ such that $g \circ h=H^{\prime \prime} \otimes \varepsilon_{H^{\prime}}$. So $H / H I\left(H^{\prime}\right) \approx\left(H^{\prime \prime} \otimes H^{\prime}\right)$ $\otimes_{H^{\prime}} k \approx H^{\prime \prime}$, and the diagram

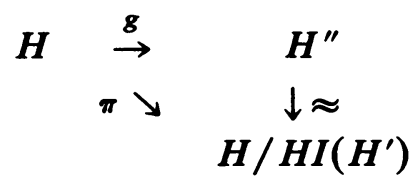

is commutative. Suppose $L \in$ obj $\mathcal{K} / k$ and $r: H \rightarrow L$ is a morphism in $\mathcal{H} / k$ such that $r \circ i=\varepsilon \eta$. Define $\bar{r}: H^{\prime \prime} \approx H / H I\left(H^{\prime}\right) \rightarrow L$ by $\bar{r}\left(h+H I\left(H^{\prime}\right)\right)=r(h)$ for $h \in H . \bar{r}$ is a well-defined morphism in $\mathcal{H} / k$ and hence $g: H \rightarrow H^{\prime \prime}$ is the cokernel of $i: H^{\prime} \rightarrow H$ in $\mathcal{H} / k$. The epimorphism $g: H \rightarrow H^{\prime \prime}$ is therefore normal.

Now suppose that $f: H \rightarrow H^{\prime \prime}$ is an epimorphism in $\mathcal{H} / k . f \mid k G(H): k G(H) \rightarrow$ $k G\left(H^{\prime \prime}\right)$ is an epimorphism; similarly $f \mid C(H): C(H) \rightarrow C\left(H^{\prime \prime}\right)$ is an epimorphism. By Proposition 4.6, $f \mid k G(H): k G(H) \rightarrow k G\left(H^{\prime \prime}\right)$ has a kernel $\zeta: k N \rightarrow k G(H)$, where $N \rightarrow G(H)$ is the kernel of $f \mid G(H): G(H) \rightarrow G\left(H^{\prime \prime}\right)$ in $\mathcal{G}$, such that $f \mid k G(H)$ is the cokernel of $\zeta$. By Proposition 4.7, $f \mid C(H): C(H) \rightarrow C\left(H^{\prime \prime}\right)$ has a kernel $\tau$ : $\tilde{H} \rightarrow C(H)$, where $\tilde{H}$ is a sub-Hopf algebra of $C(H)$ and therefore is cocomplete, such that $f \mid C(H)$ is the cokernel of $\tau$. $N$ acts on $\tilde{H}$ by conjugation, hence $k N \otimes \tilde{H}$ is a sub-Hopf algebra of $H$. Denote $k N \otimes \tilde{H}$ by $H^{\prime}$, and let $i: H^{\prime} \rightarrow H$ be the inclusion. Then $i: H^{\prime} \rightarrow H$ is the kernel of $f: H \rightarrow H^{\prime \prime}$, and $f$ is the cokernel of $i$. We thus have proved the following theorem:

4.9. ThEOREM. Every epimorphism in $\mathcal{X} / k$ is a normal epimorphism.

We conclude this section with a proposition which will enable us to carry out the constructions of $\$ 5$.

4.10. Proposition. (1) (CF. [6, Proposition 2.1]) Suppose $f: H^{\prime} \rightarrow H^{\prime \prime}$ is a

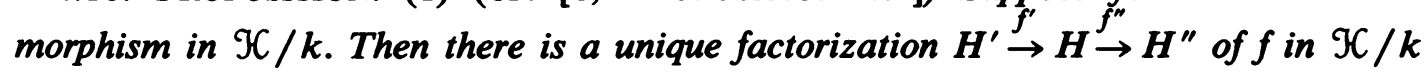
such that $f^{\prime}$ is surjective and $f^{\prime \prime}$ is injective.

(2) The category $\mathcal{H} / k$ has kernels. 
Proof. (1) Simply take $H$ to be $f\left(H^{\prime}\right)$, with $f^{\prime \prime}: H \rightarrow H^{\prime \prime}$ the natural inclusion.

(2) Suppose $f: H^{\prime} \rightarrow H^{\prime \prime}$ is a morphism in $\mathcal{H} / k$. Then by (1) $f$ has a factorization $H^{\prime} \stackrel{f^{\prime}}{\rightarrow} H \stackrel{f^{\prime \prime}}{\rightarrow} H^{\prime \prime}$ with $f^{\prime}$ surjective and $f^{\prime \prime}$ injective. By Theorem 4.8, $f^{\prime}: H^{\prime} \rightarrow H$ has a kernel $\tau: N \rightarrow H^{\prime} . \tau: N \rightarrow H^{\prime}$ is then the kernel of $f: H^{\prime} \rightarrow H^{\prime \prime}$.

5. Nilpotent objects of $\mathcal{H} / k$. As we have remarked previously, perhaps the most illuminating way to view the category $\mathcal{H} / k$ is as the category of groups in the category of supplemented split $k$-coalgebras. The principal question raised by this observation is to what extent group theoretical considerations can be applied to the category $\mathcal{K} / k$. In this section we apply the results of $\$ 4$ to define a lower central series for an object of $\mathcal{K} / k$ and we establish basic properties of nilpotent objects of $\mathcal{K} / k$.

Suppose $\mathscr{D}$ is a pointed category such that:

(i) If $s: D^{\prime} \rightarrow D^{\prime \prime}$ is a morphism in $\mathscr{D}$, then $s$ has a factorizaton $D^{\prime} \stackrel{s^{\prime}}{\rightarrow} D \stackrel{s^{\prime \prime}}{\rightarrow} D^{\prime \prime}$ in D such that $s^{\prime}$ is a normal epimorphism and $s^{\prime \prime}$ is a monomorphism.

(ii) $\mathcal{D}$ has finite products and coproducts.

In $\mathscr{D}$ the factorization of a morphism described in (i) must be unique up to a canonical isomorphism. By Proposition 4.10 we know that $\mathcal{K} / k$ is such a category.

In a category $\mathscr{D}$ as described above commutators may be defined using the following construction of Moore and Smith [6, pp. 758-761]. Let $D^{\prime}, D^{\prime \prime} \in$ obj $\mathscr{D}$.

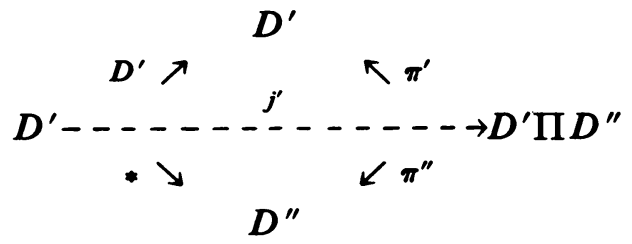

gives rise to a unique morphism $j^{\prime}$. We then have the diagram

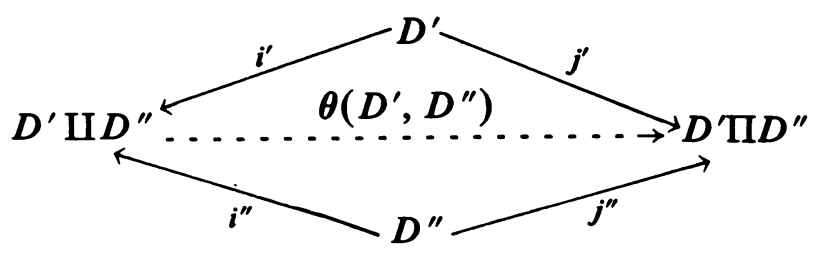

where $j^{\prime \prime}$ is constructed analogously. This gives a unique morphism $\theta\left(D^{\prime}, D^{\prime \prime}\right)$ : $D^{\prime} \amalg D^{\prime \prime} \rightarrow D^{\prime} \Pi D^{\prime \prime}$. Let $i: C\left(D^{\prime}, D^{\prime \prime}\right) \rightarrow D^{\prime} \amalg D^{\prime \prime}$ be the kernel of $\theta\left(D^{\prime}, D^{\prime \prime}\right)$. Given morphisms $f^{\prime}: D^{\prime} \rightarrow D, f^{\prime \prime}: D^{\prime \prime} \rightarrow D$ in $\mathscr{D}$, there is induced $f^{\prime} \vee f^{\prime \prime}: D^{\prime} \amalg D^{\prime \prime} \rightarrow D$. By assumption (i) on the category $\mathscr{D}$, the morphism

$$
C\left(D^{\prime}, D^{\prime \prime}\right) \stackrel{i}{\rightarrow} D^{\prime} \amalg D^{\prime \prime} \stackrel{f^{\prime} \vee f^{\prime \prime}}{\rightarrow} D
$$

has a unique factorization

$$
C\left(D^{\prime}, D^{\prime \prime}\right) \stackrel{u}{\rightarrow} \tilde{D} \stackrel{v}{\rightarrow} D
$$

with $u$ epimorphic and $v$ monomorphic. Denote $\tilde{D}$ by $\left[D^{\prime}, D^{\prime \prime}\right]_{\left(f^{\prime}, f^{\prime \prime}\right)}$. It is the commutator subobject of $D^{\prime}$ and $D^{\prime \prime}$ in $D$ with respect to the morphisms $f^{\prime}$ and $f^{\prime \prime}$.

When dealing with most algebraic structures, further restrictions are placed on the above construction. For our purposes, we only want to consider the case where 
$H^{\prime}, H^{\prime \prime} \in$ obj $\mathcal{X} / k$ such that $H^{\prime} \subseteq H$ and $H^{\prime \prime} \subseteq H$. If $i^{\prime}: H^{\prime} \rightarrow H, i^{\prime \prime}: H^{\prime \prime} \rightarrow H$ are the natural injections, then $\left[H^{\prime}, H^{\prime \prime}\right\}_{\left(i^{\prime}, i^{\prime \prime}\right)}$ is the commutator sub-Hopf algebra of $H^{\prime}$ and $H^{\prime \prime}$ in $H$. Henceforth the inclusion morphisms will be suppressed and we will write $\left[H^{\prime}, H^{\prime \prime}\right]$ for $\left[H^{\prime}, H^{\prime \prime}\right]_{\left(i^{\prime}, i^{\prime \prime}\right.}$.

An imitation of the above definition leads to the usual notions when applied to the category of groups and when applied to the category of (graded) Lie algebras over a ring $K$.

5.1. Proposition ([6, Proposition 2.6]). Let $H \in$ obj $\mathcal{K} / k$.

(1) If $H^{\prime}$ is a sub-Hopf algebra of $H$, then $H^{\prime}$ is a normal sub-Hopf algebra (i.e., the inclusion $H^{\prime} \rightarrow H$ is a normal monomorphism) if and only if $\left[H, H^{\prime}\right] \subseteq H^{\prime}$.

(2) If $H^{\prime}$ is a normal sub-Hopf algebra of $H$, then $\left[H^{\prime}, H^{\prime}\right]$ is a normal sub-Hopf algebra of $\boldsymbol{H}$.

Let $H \in$ obj $\mathcal{H} / k$ and let $H[[-1]]=[H, H]$. For any positive integer $n>1$, define $H[[-n]]$ inductively by

$$
H[[-n]]=[H, H[[-n+1]]] .
$$

We then have the lower central series for $H$ :

$$
H \supseteq H[[-1]] \supseteq \cdots \supseteq H[[-n]] \supseteq \cdots .
$$

Similarly one could define the derived series for $H$

$$
H \supseteq H[-1] \supseteq \cdots \supseteq H[-n] \supseteq \cdots
$$

by setting $H[-1]=[H, H]$ and $H[-n]=[H[-n+1], H[-n+1]]$ for $n>1$.

5.2. Defintion. A Hopf algebra $H \in \mathrm{obj} \mathcal{T} / k$ is said to be nilpotent if for some integer $n>0, H[[-n]]=k$. If $H$ is nilpotent and $n$ is the least integer such that $H[[-n]]=k, H$ is said to be nilpotent of class $n$. In this case we write $n=\operatorname{cl}(H)$.

5.3. Proposition. Suppose $H \in$ obj $\mathcal{H} / k$ is nilpotent of class $n$. Then $G=G(H)$ is a nilpotent group of class $<n$. If $\operatorname{char}(k)=0$, then $P(H)$ is a nilpotent Lie algebra of class $<n$.

Proof. If $\operatorname{cl}(H)=n$ and $H^{\prime}$ is a sub-Hopf algebra of $H$ then clearly $H^{\prime}$ is nilpotent with $\mathrm{cl}\left(H^{\prime}\right)<n$. Hence $k G$ is a nilpotent Hopf algebra with $\mathrm{cl}(k G)<n$. Since $k G \Pi k G=k G \otimes k G=k(G \times G)$, we see that the kernel of

$$
\theta(k G, k G): k(G * G)=k G * k G \rightarrow k(G \times G)
$$

is $k N \rightarrow k(G * G)$, where $N \rightarrow G * G$ is the kernel of the morphism $\theta(G, G)$ : $G * G \rightarrow G \times G$ in $\mathcal{G}$. Hence $[k G, k G]=k[G, G]$. The second assertion follows from the observation that $U\left(L^{\prime}\right) \otimes U(L)=U\left(L^{\prime} \times L\right)$ and $U\left(L^{\prime}\right) \amalg U(L)=$ $U\left(L^{\prime} \amalg L\right)$ for $L, L^{\prime}$ Lie algebras over a commutative ring $K$.

Recall that in a pointed category $\mathscr{D}$, a sequence

$$
* \rightarrow D^{\prime} \stackrel{f^{\prime}}{\rightarrow} D^{f^{\prime \prime}} \rightarrow D^{\prime \prime} \rightarrow *
$$


is called an extension if $f^{\prime}=\operatorname{Ker} f^{\prime \prime}$ and $f^{\prime \prime}=\operatorname{Coker} f^{\prime}$. From $\$ 2$ we know that if $H \in$ obj $\mathcal{K} / k$ then

$$
k \rightarrow C(H) \rightarrow H \rightarrow k G(H) \rightarrow k
$$

is an extension in $\mathcal{C} / k$.

5.4. Definition. The extension $k \rightarrow H^{\prime} \rightarrow H \rightarrow H^{\prime \prime} \rightarrow k$ in $\mathcal{H} / k$ is called central if $\left[H^{\prime}, H\right]=k$.

5.5. Proposition. Suppose $k \rightarrow H^{\prime} \rightarrow H \rightarrow H^{\prime \prime} \rightarrow k$ is an extension in $\mathcal{K} / k$. Then

(1) if $H$ is nilpotent of class $n$, then $H^{\prime}, H^{\prime \prime}$ are nilpotent with $\operatorname{cl}\left(H^{\prime}\right), \operatorname{cl}\left(H^{\prime \prime}\right)<n$.

(2) if $H^{\prime \prime}$ is nilpotent of class $n$ and the extension is central, then $H$ is nilpotent with $\operatorname{cl}(H)<n+1$.

Proof. Proceed as in Proposition 2.7 and Proposition 2.8 of [6], making the obvious necessary modifications.

5.6. Corollary. Suppose $H \in$ obj $\mathcal{H} / k$, where $k$ is a field of characteristic 0 . Also assume $[C(H), H]=k$. Then $H$ is a nilpotent Hopf algebra if and only if $G(H)$ is a nilpotent group and $P(H)$ is an abelian Lie algebra.

Proof. By assumption the extension

$$
k \rightarrow C(H) \rightarrow H \rightarrow k G(H) \rightarrow k
$$

is a central extension in $\mathcal{K} / k$. By Proposition $5.5, k G(H)$ being nilpotent implies that $H$ is nilpotent. The other implication was established in Proposition 5.3.

There are many deep results in the theory of nilpotent groups which possibly admit generalization to nilpotent Hopf algebras in $\mathcal{H} / k$. We discuss an attempt at one such generalization.

Recall that a group $G$ is called polycyclic if it has a series of subgroups

$$
G=G_{0} \supseteq G_{1} \supseteq \cdots \supseteq G_{r}=\{e\}
$$

such that, for each $i=1,2, \ldots, r, G_{i}$ is normal in $G_{i-1}$ and $G_{i-1} / G_{i}$ is cyclic. In particular, finitely generated nilpotent groups are polycyclic.

The following is a theorem of P. Hall [2, Theorem 1]:

THEOREM. If $G$ is a polycyclic group, then the integral group ring $Z G$ is left (right) Noetherian.

In an attempt to generalize this theorem to objects of $\mathcal{H C} / k$, we consider the following definitions:

5.7. Definitions. Suppose that the Hopf algebra $H$ is an element of $\mathcal{K} / k . H$ is said to be finitely generated if $H$ is finitely generated as an algebra. Similarly $H$ is said to be left (right) Noetherian if every left (right) ideal in the algebra $H$ is finitely generated.

5.8. Conjecture. Suppose $H \in$ obj $\mathcal{T} / k$ is a finitely generated nilpotent Hopf algebra. Then $H$ is left (right) Noetherian.

An examination of Hall's proof suggests the above conjecture might be proved by the following scheme. Consider the statements: 
(1) If $H \in$ obj $\mathcal{H} / k$ is finitely generated, then $H[[-n]]$ is finitely generated for all $n>0$.

(2) Suppose $k \rightarrow H^{\prime} \rightarrow H \rightarrow H^{\prime \prime} \rightarrow k$ is an extension in $\mathcal{H} / k$ such that $H^{\prime}$ and $H^{\prime \prime}$ are left (right) Noetherian. Then $H$ is left (right) Noetherian.

(3) A finitely generated abelian Hopf algebra $H \in$ obj $\mathcal{H} / k$ is Noetherian.

If (1), (2), (3) were established, Conjecture 5.8 would easily follow. For let $H \in \operatorname{obj} \mathcal{H} / k$ be a finitely generated nilpotent Hopf algebra of class $n$.

$$
k \rightarrow H[[-m]] \rightarrow H[[-m+1]] \rightarrow H[[-m+1]] / H[[-m]] \rightarrow k
$$

is an extension in $\mathcal{K} / k$ for all $m>0 . H[[-m+1]] / H[[-m]]$ is finitely generated because $H[[-m+1]]$ is finitely generated by (1). Moreover $H[[-m+1]] / H[[-m]]$ is abelian and hence is Noetherian by (3). Thus if $H[[-m]]$ is left (right) Noetherian it follows that $H[[-m+1]]$ is Noetherian. Starting with the observation that $H[[-n]]=k$ is Noetherian one can conclude that $H$ is left (right) Noetherian by the above process.

Of the statements (1), (2), (3) listed above, (3) is totally trivial. Any finitely generated commutative algebra is Noetherian as it is the homomorphic image of a polynomial algebra. The author's attempts to prove (1) and (2) were unsuccessful.

\section{REFERENCES}

1. H. Cartan and S. Eilenberg, Homological algebra, Princeton Univ. Press, Princeton, N. J., 1956.

2. P. Hall, Finiteness conditions for soluble groups, Proc. London Math. Soc. (3) 4 (1954), 419-436.

3. P. J. Hilton and U. Stammbach, A course in homological algebra, Springer-Verlag, New York, 1971.

4. J.-M. Lemaire, Algèbres connexes et homologie des espaces de lacets, Springer-Verlag, New York, 1974.

5. J. Milnor and J. C. Moore, On the structure of Hopf algebras, Ann. of Math. (2) 81 (1965), 211-264.

6. J. C. Moore and L. Smith, Hopf algebras and multiplicative fibrations. I, Amer. J. Math. 90 (1968), $752-780$.

7. M. Sweedler, Hopf algebras, Benjamin, New York, 1969.

8. O. Zariski and P. Samuel, Commutative algebra, Van Nostrand, Princeton, N. J., 1958.

Department of Mathematics, Harvard University, Cambridge, Massachusetts 02138 